Behavioral/Systems/Cognitive

\title{
Retinotopic Organization of Human Ventral Visual Cortex
}

\author{
Michael J. Arcaro, ${ }^{1,2 *}$ Stephanie A. McMains, ${ }^{1,2 *}$ Benjamin D. Singer, ${ }^{2}$ and Sabine Kastner ${ }^{1,2,3}$ \\ ${ }^{1}$ Department of Psychology, ${ }^{2}$ Center for the Study of Brain, Mind, and Behavior, and 3 Princeton Neuroscience Institute, Princeton University, Princeton, \\ New Jersey 08540
}

Functional magnetic resonance imaging studies have shown that human ventral visual cortex anterior to human visual area V4 contains two visual field maps, V0-1 and V0-2, that together form the ventral occipital (VO) cluster (Brewer et al., 2005). This cluster is characterized by common functional response properties and responds preferentially to color and object stimuli. Here, we confirm the topographic and functional characteristics of the VO cluster and describe two new visual field maps that are located anterior to V0-2 extending across the collateral sulcus into the posterior parahippocampal cortex (PHC). We refer to these visual field maps as parahippocampal areas PHC-1 and PHC-2. Each PHC map contains a topographic representation of contralateral visual space. The polar angle representation in PHC-1 extends from regions near the lower vertical meridian (that is the shared border with V0-2) to those close to the upper vertical meridian (that is the shared border with PHC-2). The polar angle representation in PHC-2 is a mirror reversal of the PHC-1 representation. PHC-1 and PHC-2 share a foveal representation and show a strong bias toward representations of peripheral eccentricities. Both the foveal and peripheral representations of PHC-1 and PHC-2 respond more strongly to scenes than to objects or faces, with greater scene preference in PHC-2 than PHC-1. Importantly, both areas heavily overlap with the functionally defined parahippocampal place area. Our results suggest that ventral visual cortex can be subdivided on the basis of topographic criteria into a greater number of discrete maps than previously thought.

\section{Introduction}

In the human brain, objects are represented in a large swath of ventral temporal and lateral occipital cortex that responds more strongly to object stimuli than to their scrambled counterparts (Malach et al., 1995; Grill-Spector et al., 1999; Kourtzi and Kanwisher, 2001) (for review, see Grill-Spector and Malach, 2004). Initially, it was thought that this substantial part of visual cortex had no additional functional organization other than containing a small number of modules that appear to preferentially process information regarding specific biologically relevant object categories such as faces [the fusiform face area (Kanwisher et al., 1997)], places [the parahippocampal place area (PPA) (Epstein and Kanwisher, 1998)], and bodies [the extrastriate body area (Downing et al., 2001) and the fusiform body area (Peelen and Downing, 2005)]. This notion was later revised by Malach and colleagues who found evidence for a systematic representation of eccentricity in ventral visual cortex, with medial regions, such as parahippocampal cortex, representing peripheral eccentricities and lateral regions, such as lateral occipital cortex, representing foveal eccentricities (Levy et al., 2001; Hasson et al., 2002, 2003; Malach et al., 2002). However, no orderly representation of polar angle was found to delineate visual areas by

\footnotetext{
Received June 15, 2009; revised July 9, 2009; accepted July 20, 2009.

This work was supported by National Institutes of Health Grant R01EY-017699. We thank J. D. Swisher for helpful comments and for providing code for the retinotopy stimuli, M. V. Peelen for object category stimuli and helpful comments, and Z. Saad for help with surface analysis.

*M.J.A. and S.A.M. contributed equally to this study.

Correspondence should be addressed to Sabine Kastner, Department of Psychology, Green Hall, Princeton

University, Princeton, NJ 08540. E-mail: skastner@princeton.edu.

DOI:10.1523/JNEUROSCI.2807-09.2009

Copyright $\odot 2009$ Society for Neuroscience $\quad$ 0270-6474/09/2910638-15\$15.00/0
}

meridian boundaries, an organization that is typical for early visual cortex (Levy et al., 2001). According to the organizing principle of eccentricity, object categories requiring foveal vision, such as faces and words, are associated with center-biased representations, whereas objects that require the integration of parts over a larger scale, such as scenes and buildings, are associated with periphery-biased representations (Hasson et al., 2002).

More recently, however, improved imaging techniques at higher field strength have revealed an increasing number of retinotopically organized areas containing both an orderly polar angle as well as an eccentricity representation in ventral visual cortex. Anterior to human visual area V4 (hV4), in ventral occipital (VO) cortex, two visual field maps have been found that share a fovea, and each contain a topographic representation of contralateral visual space, termed VO- 1 and VO-2 (Brewer et al., 2005). The VO areas are characterized by similar response profiles and code for stimulus color and object-related information. In lateral occipital (LO) cortex, two areas have been identified that share a fovea with early visual cortex and hV4, and each contain a representation of contralateral space, termed LO-1 and LO-2 (Larsson and Heeger, 2006). As with the VO areas, the LO areas appear to share common functional response properties and code for stimulus shape.

Using attentive wedge and ring tracking paradigms as well as standard phase-encoded retinotopic mapping, we confirm the organization of $\mathrm{hV} 4$ and the $\mathrm{VO}$ areas and report here two new visual field maps anterior to $\mathrm{VO}-2$ within posterior parahippocampal cortex (PHC), referred to as PHC-1 and PHC-2. The $\mathrm{PHC}$ areas were found to share a fovea, represent contralateral space, and show a strong bias toward the representation of peripheral eccentricities. Both the foveal and peripheral representations of PHC-1 and PHC-2 responded more strongly to scenes 
than to objects or faces and heavily overlapped with the functionally defined PPA. Our findings lend additional support to the notion that object-related cortex is parcellated into multiple retinotopically organized areas (Wandell et al., 2007).

\section{Materials and Methods}

\section{Subjects}

Eleven subjects (aged 20-36 years, four females) participated in the study, which was approved by the Institutional Review Panel of Princeton University. All subjects were in good health with no history of psychiatric or neurological disorders and gave their informed written consent. Subjects had normal or corrected-to-normal visual acuity. All subjects participated in three scanning sessions, during which highresolution structural images were acquired for cortical surface reconstructions, and polar angle and eccentricity measurements were obtained across visual cortex using attentive wedge and ring tracking paradigms (attentionotopy studies). Three subjects exhibited excessive head motion and were excluded from additional analyses. The remaining eight subjects participated in a fourth scanning session in which various object stimuli were probed, and four of them participated in a fifth and sixth scanning session, in which polar angle and eccentricity maps were measured using standard retinotopic mapping procedures (Sereno et al., 1995; DeYoe et al., 1996; Engel et al., 1997).

\section{Visual display}

The stimuli were generated on Macintosh G4 and G5 computers (Apple Computers) using MATLAB software (MathWorks) and Psychophysics Toolbox functions (Brainard, 1997; Pelli, 1997). Stimuli were projected from a PowerLite 7250 liquid crystal display projector (Epson) located outside the scanner room onto a translucent screen located at the end of the scanner bore. Subjects viewed the screen at a total path length of 60 $\mathrm{cm}$ through a mirror attached to the head coil. The screen subtended $30^{\circ}$ of visual angle in both the horizontal and vertical dimensions. A trigger pulse from the scanner synchronized the onset of stimulus presentation to the beginning of the image acquisition.

\section{Visual stimuli and experimental design}

Polar angle measurements. To measure polar angle representations in visual cortex, visual stimuli consisted of a wedge that rotated either clockwise or counterclockwise around a central fixation point (supplemental Fig. $1 \mathrm{~A}$, available at www.jneurosci.org as supplemental material). The wedge spanned $1-15^{\circ}$ in eccentricity with an arc length of $45^{\circ}$ and moved at a rate of $9 \%$. There were two variations of wedge stimuli, one used in the attentionotopy studies and one used in the standard retinotopic mapping studies.

The wedge used in the attentionotopy studies was filled with 1000 white dots $\left(0.1^{\circ}, 65 \mathrm{~cd} / \mathrm{m}^{2}\right)$ that moved either randomly or in a coherent direction at a rate of $7 \%$ s. The percentage of coherently moving dots ranged from 30 to $65 \%$ and was determined separately for each subject based on the individual motion coherence threshold to yield behavioral performance of $\sim 75 \%$ accuracy in a behavioral testing session before scanning. The direction of motion for the coherent dots changed randomly every $3-5 \mathrm{~s}$. Subjects were instructed to maintain fixation while covertly attending to the rotating wedge and to detect a change in the direction of the coherently moving dots by pressing a button with their right index finger. The change in radial direction of the coherently moving dots ranged between $75^{\circ}$ and $105^{\circ}$. Each run consisted of six cycles of $40 \mathrm{~s}$ each of the rotating wedge and started and ended with a $10 \mathrm{~s} \mathrm{blank}$ period, amounting to an overall run length of $260 \mathrm{~s}$. Runs alternated between clockwise and counterclockwise wedge rotations, with a total of 10 runs per scan session.

The wedge used for the standard retinotopic mapping studies consisted of a colored checkerboard, with the chromaticity and luminance of each check alternating at a flicker frequency of $4 \mathrm{~Hz}$ (supplemental Fig. $1 \mathrm{~A}$, available at www.jneurosci.org as supplemental material) (for details, see Swisher et al., 2007). A transparent wedge within a dark foreground rotated around a central fixation. The underlying checkerboard was only visible through the transparent wedge, giving the appearance of a rotating checkerboard wedge. The size and speed of the rotating wedge was the same as in the attentionotopy experiment. Subjects attended to and performed a luminance detection task at fixation, indicating by button press when a change in luminance occurred. Luminance changes occurred on average every $4.5 \mathrm{~s}$. Each run consisted of seven cycles of the rotating checkerboard. Runs alternated between clockwise and counterclockwise wedge rotation, with a total of 10 runs per scan session.

Eccentricity measurements. To measure eccentricity representations in visual cortex, visual stimuli consisted of an annulus that either expanded or contracted around a central fixation point (supplemental Fig. $1 B$, available at www.jneurosci.org as supplemental material). The duty cycle of the annulus was $12.5 \%$, that is, any given point on the screen was within the annulus for only $12.5 \%$ of the time. The annulus increased on a logarithmic scale over time in size and rate of expansion to approximately match the human cortical magnification factor of early visual cortex (Horton and Hoyt, 1991; Swisher et al., 2007). The outer part of the ring expanded to a maximum eccentricity of $16.875^{\circ}$, to ensure that the whole visual display $\left(1-15^{\circ}\right)$ was stimulated for an equal amount of time, before returning to the foveal origin (and vice versa for the contracting ring). There were two variations of ring stimuli, one used in the attentionotopy studies and one used in the standard retinotopic mapping studies.

The ring used in the attentionotopy studies was red $\left(9.8 \mathrm{~cd} / \mathrm{m}^{2}\right)$ and filled with white balls $\left(65 \mathrm{~cd} / \mathrm{m}^{2}\right)$ bouncing randomly within the annulus. The diameter of each ball varied such that the radius was always equal to one-quarter of the ring size throughout the cycle. Additionally, the number of balls varied (15-20) throughout each cycle to maintain full coverage within the annulus. Every 3-5 s, one ball, that was randomly chosen, changed in luminance. The presentation length of the luminance change was matched to each subject's detection threshold to yield behavioral performance of $\sim 75 \%$ accuracy, as determined in behavioral testing sessions before scanning, and ranged from 0.08 to $0.15 \mathrm{~s}$. During each run, subjects were instructed to maintain fixation while covertly attending to the bouncing balls within the ring and to perform the luminance detection task. The stimulus was coded to allow the balls to move about freely within the ring. Each run consisted of six cycles of $40 \mathrm{~s}$ each and started and ended with a $10 \mathrm{~s}$ blank period amounting to an overall run length of $260 \mathrm{~s}$. Runs alternated between annulus expansions and contractions, with a total of 10 runs per scan session.

The ring used for the standard eccentricity mapping consisted of a colored checkerboard, with the chromaticity and luminance of each check alternating at a flicker frequency of $4 \mathrm{~Hz}$ (supplemental Fig. $1 \mathrm{~B}$, available at www.jneurosci.org as supplemental material) (for details, see Swisher et al., 2007). A transparent annulus within a dark foreground layer either expanded or contracted around a central fixation point. The underlying checkerboard was only visible through the transparent annulus, giving the appearance of an expanding or contracting checkerboard ring. Subjects maintained fixation and performed a central luminance detection task at fixation, as described in the last section. The size and rate of expansion for the ring was the same as in the attentionotopy experiment. Each run consisted of seven cycles of $40 \mathrm{~s}$ each, and runs alternated between annulus expansion and contraction, with a total of 10 runs per scan session.

Object stimuli. To determine functional response properties with respect to object-related information across ventral visual cortex, object stimuli from the following categories were used: faces, scenes, inanimate objects, headless bodies, houses, flowers, chairs, tools, and scrambled stimuli (Downing et al., 2006). For the current study, analyses were limited to face, body, inanimate object, scene, and scrambled stimuli. For each category, the stimulus pool contained 40 different grayscale images (supplemental Fig. 1C, available at www.jneurosci.org as supplemental material). Each object stimulus subtended $12^{\circ}$ horizontally by $12^{\circ}$ vertically and was presented behind a central fixation point $\left(0.3^{\circ}, 0.9 \mathrm{~cd} / \mathrm{m}^{2}\right)$ for $350 \mathrm{~ms}$, followed by a $400 \mathrm{~ms}$ blank period. Twenty images from a given category were shown in a block that lasted for $15 \mathrm{~s}$. Subjects performed a one-back task indicating the repeated appearance of a stimulus, which occurred randomly three times within each block. During a given run, two blocks from each category were shown in pseudorandomized order, amounting to a total of 18 presentation blocks per condition. A fixation block of $15 \mathrm{~s}$ without stimulus presentations was interleaved 
after every four blocks of object stimuli presentations, resulting in an overall run length of $345 \mathrm{~s}$. Four to six runs were tested during a scan session.

\section{Data acquisition}

Data were acquired with a 3T Allegra head-dedicated magnetic resonance imaging (MRI) scanner (Siemens) using a four-channel bitemporal phased array coil (model NMSC-003A; Nova Medical). For the attentionotopy and standard retinotopy studies, 20 axial slices were acquired in 10 runs of 130 and 140 volumes, respectively, covering ventral occipital and temporal cortex. For the object category studies, 25 axial slices were acquired in four to six runs of 138 volumes, covering ventral occipital and temporal cortex. All acquisitions used a gradient echo, echo planar sequence with a 128 square matrix (slice thickness of $2 \mathrm{~mm}$, with a $0.5 \mathrm{~mm}$ gap between slices, interleaved acquisition), leading to an inplane resolution of $2 \times 2 \mathrm{~mm}^{2}$ [field of view (FOV), $256 \times 256 \mathrm{~mm}^{2}$; repetition time (TR), 2.0-2.5 s; echo time (TE), $40 \mathrm{~ms}$; flip angle, $90^{\circ}$ ]. A partial Fourier factor of $7 / 8$ was used to acquire asymmetric fraction of $k$-space to reduce the acquisition time. Echo planar images were compared with a high-resolution anatomical scan taken at the end of each session [magnetization-prepared rapid-acquisition gradient echo (MPRAGE) sequence; TR, $2.5 \mathrm{~s}$; TE, $4.38 \mathrm{~s}$; flip angle, $8^{\circ}$; $256 \times 256$ matrix; $1 \mathrm{~mm}^{3}$ resolution)]. An in-plane magnetic field map image was acquired to perform echo planar imaging undistortion (FOV, $256 \times 256 \mathrm{~mm}$; 128 matrix; TR, $345 \mathrm{~ms}$; TE, 5.06/8.06 ms; flip angle, $40^{\circ}$; bandwidth, $260 \mathrm{~Hz} /$ pixel). For cortical surface reconstructions, high-resolution structural scans were acquired in a separate session (MPRAGE sequence, same parameters as above, two acquisitions).

\section{Data analysis}

Data were analyzed using AFNI (Cox, 1996) (http://afni.nimh.nih.gov/ afni/), SUMA (http://afni.nimh.nih.gov/afni/suma/), MATLAB, and FreeSurfer (Dale et al., 1999; Fischl et al., 1999) (http://surfer.nmr.mgh. harvard.edu/). Functional images were motion corrected (Cox and Jesmanowicz, 1999) to the image acquired closest in time to the anatomical scan, undistorted using the images from the field map scan, and normalized to percentage signal change by dividing the time series by its mean intensity. After normalization, attentionotopy and retinotopy data were projected onto cortical surface reconstructions created with FreeSurfer that were aligned to each of the experimental sessions using AFNI/ SUMA. All voxels that fell between the gray and white matter boundaries were mapped to the surface. The units of data projected to the surface are referred to as nodes. Given that there is not a one-to-one correspondence between nodes and voxels, the value of each node was calculated by taking a weighted average of all the voxels that overlapped with each node. All subsequent analysis procedures for attentionotopy and retinotopy datasets (e.g., Fourier analysis) were performed on the surfacemapped data, which favorably restricts analyses to data that are primarily within the gray matter, because white matter voxels do not get mapped onto the surface. No spatial smoothing was used for any of the analyses (i.e., topography and object representations). For display purposes, the attentionotopy and retinotopy data presented in the figures were smoothed with a $2 \mathrm{~mm}$ full-width at half-maximum on the surface (Chung et al., 2005). For each subject, structural images were transformed into Talairach space and linked to the surface reconstructions using AFNI software to obtain Talairach coordinates for the areas investigated (Talairach and Tournoux, 1988). Surface size estimates were measured using SUMA tools that calculate the volume of all nodes that fall between the white matter and pial surface layers.

For each attentionotopy dataset, the volumes acquired during the blank periods were discarded. For each retinotopy dataset, the first 20 volumes corresponding to the first cycle were discarded. A Fourier analysis was used to identify surface nodes activated by the polar angle and eccentricity stimuli (Bandettini et al., 1993; Engel et al., 1994). For each node of the surface, the amplitude and phase-the temporal delay relative to the stimulus onset-of the harmonic at the stimulus frequency (SF) was determined by a Fourier transform of the mean time series of the node. To correctly match the phase delay of the time series of each node to the phase of the wedge/ring stimuli, and thereby localize the region of the visual field to which the underlying neurons responded best, the response phases were corrected for the hemodynamic lag $(3 \mathrm{~s})$. The counterclockwise/inward runs were then reversed to match the clockwise/ outward runs and averaged together for each node. An $F$ ratio was calculated by comparing the power of the complex signal at the stimulus frequency with the power of the noise. From the $F$ ratio, we calculated a $p$ value (uncorrected) taking into account degrees of freedom of the signal and noise. To quantify the reliability of phase estimates across runs, the variance of a mean phase across cycles was determined for each node. A jackknifing method in which phase estimates were calculated from $n-2$ cycles (eliminating one clockwise and one counterclockwise cycle per calculation) across all runs was used to determine the SE of phase estimates (for similar application, see Hansen et al., 2007). A grand mean phase estimate was calculated from the average of each of these phase estimates along with the SE to account for variance across estimates for each node. The SE was then converted into seconds per cycle.

Statistical maps were thresholded at a variance of $1.5 \mathrm{~s}$ of the $40 \mathrm{~s}$ cycle and overlaid on cortical surface reconstructions. The pattern and significance of activation approximately compares with a statistical threshold of $p<0.01$ (uncorrected for multiple comparisons, derived from the $F$ ratio that was calculated from the Fourier transform). When displaying phase estimates, a 12 point color scale was assigned to the polar angle datasets with each color representing $18^{\circ}$ visual angle, and a 10 point color scale was assigned to the eccentricity datasets with each color representing $1.5^{\circ}$ eccentricity. For the purpose of this report, we focused our analysis on ventral temporal cortex, i.e., to brain regions located along the collateral sulcus and adjacent cortex. Contiguous clusters of activated nodes within this anatomical region that showed a systematic representation of visual space in polar or eccentricity coordinates were defined as regions of interest (ROIs). Borders between adjacent visual areas were defined as reversals in polar phase progression of the contralateral visual field. Surface analyses of these ROIs were performed using AFNI and MATLAB. To estimate the representation of the visual field for each ROI, the polar angle and eccentricity phases for each surface node were plotted, including only nodes that had both significant values for polar angle and eccentricity measures. Nodes that only had a significant polar angle representation but no significant eccentricity measurement (or vice versa) were not included in the plots. To compute the representation of the visual field for each visual area as a function of polar angle and eccentricity, the visual field was divided into several sectors: contralateral and ipsilateral, upper and lower, and foveal $\left(0-7.5^{\circ}\right)$ and peripheral (7.5$\left.15^{\circ}\right)$. The number of nodes within each sector was tallied and divided by the total number of nodes in each area to derive a mean representation for each subject. Data were collapsed across hemispheres and averaged across subjects to derive a group mean average. $t$ tests were used to assess statistical significance.

To quantitatively compare the alignment between the attentionotopy and retinotopy datasets for each subject, we calculated alignment indices and correlation coefficients for each visual area. The alignment index (AI) was defined as follows: Alignment Index $=1-|\Delta \phi| / \pi$, where $\Delta \phi$ is the difference between the polar angle (or eccentricity) phase for the two experiments (for additional details, see Sereno and Huang, 2006). Only nodes that had phase values for both datasets thresholded at $1.5 \mathrm{~s}$ variance were included in the analysis. AIs were calculated for both polar angle and eccentricity measurements within each ROI on a node-bynode basis. The distribution of AI values within an ROI were plotted for single subjects in a histogram (see Fig. 7A). AI distributions peaking at or near 1 indicate that the two datasets were in good alignment (indicating that the polar angle at a vertex is identical in the two datasets). In contrast, for two uncorrelated datasets, the distribution of AIs is a shallow linear ramp starting at a count of 0 at an $\mathrm{AI}$ of 0 and ending at a small value ( $2 v / n$, where $v$ is the number of vertices, and $n$ is the number of bins) at an AI of 1 (see Fig. 7A, red line) (Sereno and Huang, 2006). To perform statistical comparisons on the AIs, single subject mean index values were derived by averaging across all index values obtained for individual nodes within an ROI. One-sample $t$ tests were conducted for each ROI between the mean index values and an index alignment value of 0.5 (representing chance). To derive a group index value, single subject mean index values were averaged within each ROI. To further evaluate the strength of align- 
ment between both datasets for a given ROI, the correlation between phase estimates was calculated on a node-by-node basis for each subject.

For the studies on object representations, data analysis was performed within the volume. Square-wave functions matching the time course of the experimental design were convolved with a gamma-variate function (Cohen, 1997) and used as regressors of interest in a multiple regression model (Friston et al., 1995). Additional regressors were included in the regression model to account for variance attributable to baseline shifts between time series, linear drifts within time series, and head motion. Beta-weights corresponding to the amplitude of the gamma function were extracted for each condition and for each node and were scaled to mean percentage signal change values. Activations were projected onto each subject's cortical surface, and the ROIs defined for each hemisphere on the basis of topographic criteria were overlaid. The mean percentage signal change for each condition was averaged across all nodes within a given ROI that were activated by the current stimuli as defined by the contrast of all visual presentation blocks versus fixation, thresholded at $p<0.0001$, uncorrected for multiple comparisons. These data were further quantified by defining a scene preference index (SPI). The scene preference index quantifies the response preference of an area evoked by scene stimuli relative to those evoked by inanimate object stimuli [SPI = ( $\left.R_{\text {scene }}-R_{\text {object }}\right) /\left(R_{\text {scene }}+R_{\text {object }}\right)$, where $R$ is average percentage signal change within an ROI]. Positive index values indicate preference for scene stimuli, values around 0 indicate no preference, and negative vales indicate preference for inanimate object stimuli. ANOVAs were used to assess statistical significance of percentage signal change within and across cortical areas hV4, VO-1, VO-2, PHC-1, and PHC-2. Data were collapsed across hemispheres for additional analysis, because no hemispheric differences were found for any of the ROIs for the different object conditions. Two-tailed $t$ tests were used to assess statistical significance of object responses for both index values and mean percentage signal change within each cortical area and for the behavioral data. Brain regions responding preferentially to scenes were identified by contrasting presentation blocks of scenes with inanimate objects $(p<0.0001$, uncorrected for multiple comparisons). This yielded a contiguous cluster of bilateral activations within the collateral sulcus in posterior parahippocampal cortex (Epstein et al., 1999). Brain regions responding preferentially to faces than to other objects were identified by contrasting presentation blocks of faces with inanimate objects $(p<0.0001$, uncorrected for multiple comparisons) (Kanwisher et al., 1997; Haxby et al., 1999).

\section{Eye-movement recordings}

For the attentionotopy experiments, eye movements were monitored for each subject in behavioral testing sessions outside the scanner. Subjects placed their heads on a chinrest located $60 \mathrm{~cm}$ in front of a monitor (Mitsubishi Electronics America), while performing the same task as used during the scanning sessions. Eye position was measured at a sampling rate of $60 \mathrm{~Hz}$ and was displayed in real time on a video monitor, superimposed on the stimulus image using a telephoto lens (model 5000 control unit and standard model 504 remote optics; Applied Science Laboratories). The experimenter observed the eye-position display to ensure that the subjects were alert and maintained central fixation. Eyeposition data were recorded for four subjects on the stimulus computer through a serial interface with the eye-tracker control module. The eyetracking system had a resolution of $0.14^{\circ}$ and the ability to resolve differences in relative eye position of $0.25^{\circ}$ or less. Ilab software (Gitelman, 2002) was used to analyze the eye-movement data. Data were processed to automatically detect and remove eye blinks. Subjects maintained fixation within a $2.5^{\circ}$ window for $97 \pm 0.01 \%$ (SEM) of the time in the polar angle experiment and $97 \pm 0.03 \%$ in the eccentricity experiment, indicating an excellent ability to maintain fixation while covertly directing attention to the peripheral target stimuli.

\section{Results}

Polar angle and eccentricity maps in ventral visual cortex

The polar angle component of retinotopic maps in ventral visual cortex was measured for the central $15^{\circ}$ of the visual field using a smoothly rotating wedge stimulus that was filled with moving dots (supplemental Fig. 1A, available at www.jneurosci.org as supplemental material). Subjects were instructed to maintain fixation while covertly attending to the rotating wedge stimulus and to detect a change in the direction of the coherently moving dots. Subjects performed at an accuracy of $73 \pm 0.03 \%$ (SEM) on average during the scan sessions. The behavioral data were further evaluated relative to the location of the wedge stimulus in the visual field. There were no significant performance differences across the visual field (supplemental Fig. $2 A$, available at www. jneurosci.org as supplemental material $)\left(F_{(19,38)}=0.442 ; p>\right.$ $0.05)$. In addition, there were no significant differences in performance for runs with clockwise compared with counterclockwise wedge rotation $\left(F_{(1,2)}=0.494 ; p>0.05\right)$.

The eccentricity component of the topographic maps in ventral visual cortex was measured for the central $15^{\circ}$ of the visual field using an annulus, filled with bouncing balls that either expanded or contracted around a central fixation point (supplemental Fig. $1 B$, available at www.jneurosci.org as supplemental material). Subjects were instructed to maintain fixation while covertly attending to the annulus and to detect a luminance change that occurred randomly in one of the balls. Subjects performed at an accuracy of $80 \pm 0.01 \%$ on average. Performance was similar across the visual field (supplemental Fig. $2 B$, available at www.jneurosci.org as supplemental material), and an ANOVA showed no significant differences in performance as a function of eccentricity $\left(F_{(12,84)}=0.639 ; p>0.05\right)$.

Bilateral activations within visual cortex extending from the calcarine sulcus, across the collateral sulcus, and into the posterior PHC were found in all eight subjects in the polar angle and eccentricity mapping studies. For the polar angle measurements, activations within each hemisphere were mainly confined to the contralateral hemifield. Individual activation maps of polar angle and eccentricity in ventral visual cortex are shown overlaid on flattened surface reconstructions for two representative subjects (S1 and S2) in Figures 1 [right hemisphere (RH)] and 2 [left hemisphere (LH)]. Additional activation maps are shown in Figure $7 A$ for subject $S 3$ and in supplemental Figures 3-5 for subjects S3-S8 (available at www.jneurosci.org as supplemental material). For each surface node, the variance of the phase estimates across runs was calculated using a jackknifing method (see Materials and Methods), and the threshold was chosen to only include data with a variance of $<1.5$ s per 40 s cycle. The color of each surface node was determined by the phase of its response and indicates the region of the visual field to which the surface node was most responsive. For the polar angle component, the upper visual field (UVF) is denoted in red-yellow, the horizontal meridian (HM) in green, and the lower visual field (LVF) in blue. Area boundaries that are formed by phase angles at or close to either the upper (UVM) or lower (LVM) vertical meridian are indicated with dotted and dashed lines, respectively. For the eccentricity measurements, the fovea is denoted in red and the periphery in blue.

We consistently found eight distinct topographically organized cortical areas within ventral occipital and temporal cortex in each subject. Six of these areas have been reported previously: V1v, V2v, V3v, hV4, VO-1, and VO-2 (Sereno et al., 1995; DeYoe et al., 1996; Engel et al., 1997; Tootell et al., 1997; Brewer et al., 2005). Anterior to VO-2, we identified two additional areas in the posterior portion of the PHC, which we will refer to as PHC-1 and PHC-2 in keeping with a labeling scheme that emphasizes anatomical landmarks rather than function or presumed homology to the macaque cortex (for similar approaches, see Brewer et 
al., 2005; Larsson and Heeger, 2006; Wandell et al., 2007). All eight areas are described in greater detail below.

Areas V1, V2, and V3

Consistent with numerous previous studies (Sereno et al., 1995; DeYoe et al., 1996; Engel et al., 1997), ventral areas V1, V2, and V3 were identified in all subjects $(n=$ 8) from a phase progression starting in the calcarine sulcus from an HM representation (green, not marked) to a UVM representation (red, dotted) that forms the border to area V2, and then reversing back to a HM that corresponds to the border of ventral V2 and V3 (Figs. 1, 2, left panels). The anterior border of ventral V3 was formed by a representation of the UVM. These three areas represent the upper quadrant of the contralateral visual field. Ventral V1, V2, and V3 share a foveal confluence; the peripheral representations extend toward the collateral sulcus (Figs. 1, 2, right panels).

Area hV4

Adjacent and anterior to ventral V3, a representation of contralateral space was identified that extended along the ventral surface and shared its posterior border, an UVM, with ventral V3 (Figs. 1, 2, left panels, red, dotted line), whereas its anterior border was formed by LVF angles (blue, dashed line). All subjects showed the same general topographic organization of hV4 in both hemispheres. For additional examples, see supplemental Figures 3-5 (available at www. jneurosci.org as supplemental material). The fovea of hV4 was found to be continuous with the foveal confluence shared by ventral V1, V2, and V3 (Figs. 1, 2, right panels), and the peripheral representation extended toward the collateral sulcus, parallel with the eccentricity map of ventral V1, V2, and V3. As observed previously (Hansen et al., 2007), the anterior border of hV4 was often formed by a continuous representation of LVF angles, such as the one seen in the RH of subject S1 (Fig. 1, left panel, 7 of 16 hemispheres), but was sometimes formed by a discontinuous representation of LVF angles intermixed with those closer to the HM representation, as seen in the RH of subject S2 (Fig. 1, left panel, 9 of 16 hemispheres). The medial part of this border was drawn in the region of the most peripheral representations found in hV4 (purple line) that reversed from there toward the foveal representations of VO-1. Together, our data suggest a representation of contralateral space anterior to ventral V3 that is consistent with the hV4 model, proposed by Wade et al. (2002) (see also Kastner et al., 1998, 2001). This mapping scheme has been termed human $\mathrm{V} 4$, or hV4, to distinguish it from the topography observed in macaque V4 that is different and represents only a quarter field (Gattass et al., 1988; Wade et al., 2002; Brewer et al., 2005) (see also Kastner et al., 1998, 2001).

In a complementary approach to investigate the topographic organization of hV4, its polar phase progression was quantita- tively evaluated. Small line segments were successively drawn parallel to the polar angle progression and perpendicular to the eccentricity progression from the posterior border of hV4 to the anterior border of PHC-2, as indicated by the schematic outlines in Figure $3 A$. The blue dots indicate the phase values for individual nodes located along the line segments, and the red line indicates the average phase values as a function of distance on the surface. Individual subject polar phase progressions between area borders were interpolated into a common space, which allowed for group averaging (Fig. 3B). As seen in the LH of subject S1 (Fig. $3 A)$ as well as the group polar phase plots for both hemispheres (Fig. $3 B$ ), the anterior and posterior borders of hV4 corresponded to the peaks of phase angles near the LVM and UVM, respectively, with a smooth progression of phase values between. The mean Talairach coordinates for left and right hV4 were $-23,-75,-11$ and $+26,-77,-11$ (Table 1 ). The mean activation sizes of hV4, for the RHs and LHs, were $1410 \pm 329$ and $1152 \pm 289 \mathrm{~mm}^{3}$ respectively, which is 50 and $46 \%$ of the surface area for the RH and LH of V1 (Table 2, Fig. 4).

\section{VO- 1 and $V O-2$}

In accordance with Brewer et al. (2005), two representations of contralateral space, VO-1 and VO-2, were identified anterior to hV4 in all subjects. These two areas were located along the pos- 

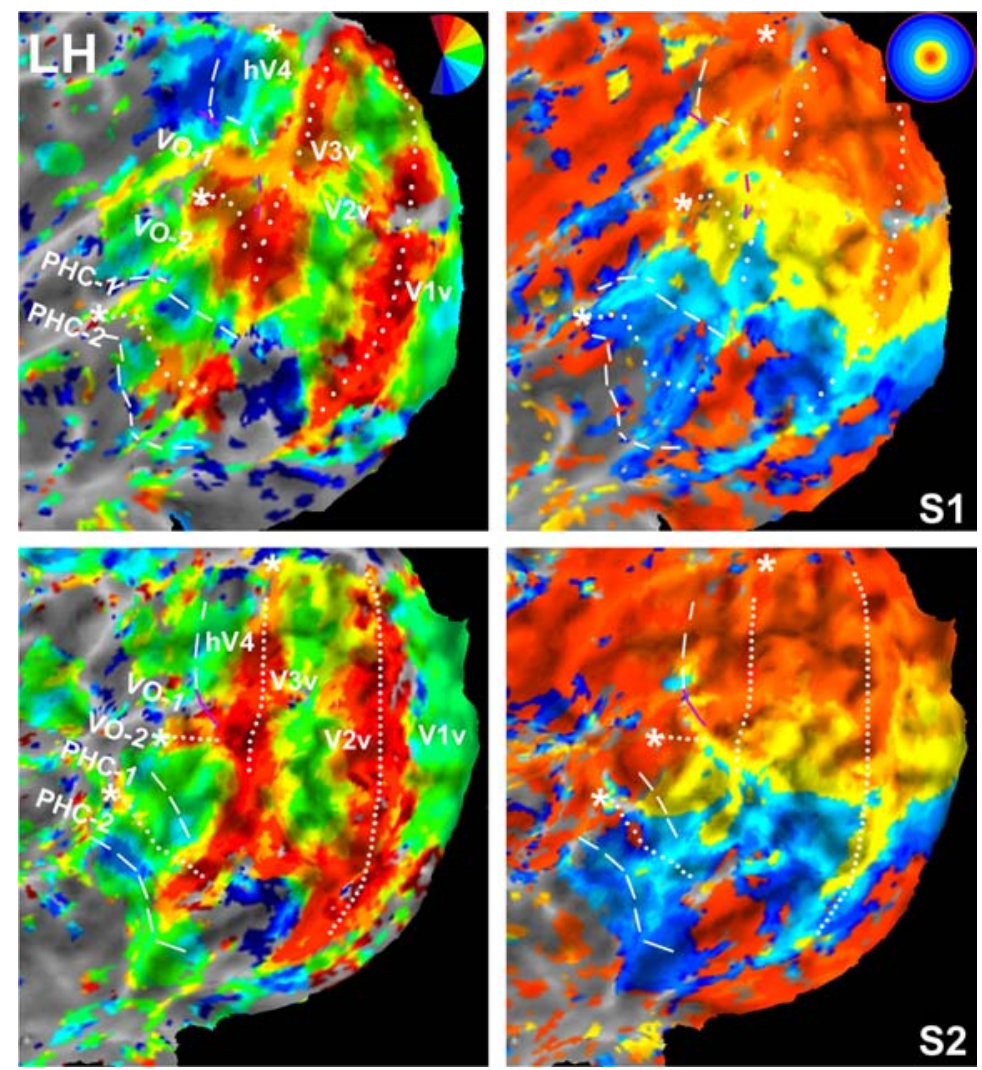

Figure 2. Polar angle and eccentricity maps in human ventral visual cortex obtained in attentionotopy studies: left hemisphere. Flattened surface reconstructions of early and ventral visual cortex of the same subjects (S1 and S2) shown in Figure 1. All conventions as in Figure 1.

terior medial fusiform gyrus and within the posterior portion of the collateral sulcus. The posterior extent of VO- 1 shared a border with hV4 that was constituted by a polar angle phase reversal within the LVF near the LVM (Figs. 1, 2, left panels, blue, dashed line). VO-1 and VO-2 shared a border that constituted UVF angles close to the UVM (red, dotted line), and the anterior border of VO-2 was formed by LVF angles toward the LVM. Similar to the anterior border of hV4, the border shared by VO-1 and VO-2 was often formed by a continuous representation of visual field angles near the UVM, such as in the LH of subjects S1 and S2 (Fig. 2, left panel, 10 of 16 hemispheres), but was sometimes formed by phase angle representations near the UVM intermixed with those closer to the HM representation as seen in the RH of subject S3 (see Fig. 7A, 6 of 16 hemispheres). Likewise, the anterior border of VO-2 was sometimes formed by a continuous representation of phase angles close to the LVM ( 5 of 16 hemispheres) but was often intermixed with those closer to the HM representation as seen in the RH of subject S2 (Fig. 1, left panel, 11 of 16 hemispheres). The mirror reversal in phase angle representations within VO-1 and VO-2 is further illustrated by the pattern of phase progressions (Fig. 3). A foveal representation separate from the large foveal confluence shared by V1, V2, V3, and hV4 (Figs. 1, 2, right panels, asterisk) was identified that was typically shared by VO-1 and VO-2 and located near the border between VO-1 and VO-2 along the posterior part of the medial fusiform gyrus. The periphery of the visual field was represented posterior from the foveal representation abutting the peripheral representations of hV4 and ventral V3. The lateral border of VO- 1 was identified as extending from the VO fovea to the peripheral extent abutting hV4 (purple line). In 12 of the 16 hemi- spheres, the foveal representation was evenly split between the two areas, as is the case for the LH of subject S2 (Fig. 2, right panel, asterisk). In the remaining cases, the foveal representations were primarily located within VO-2 with only a small part extending into $\mathrm{VO}-1$, as in the $\mathrm{RH}$ of subject S1 (Fig. 1, right panel, asterisk).

Mean Talairach coordinates for left and right $\mathrm{VO}-1$ were $-27,-69,-8$ and $+27,-67,-8$, respectively, and for left and right VO-2 were $-26,-60,-7$ and +25 , $-60,-7$, respectively (Table 1 ). The mean activation sizes of VO-1 and VO-2 were 1145 and $1452 \mathrm{~mm}^{3}$ respectively, which is 22 and $27 \%$ the size of $\mathrm{V} 1$ and 45 and $57 \%$ the size of hV4 (Table 2, Fig. 4).

\section{PHC- 1 and $P H C-2$}

Two cortical areas, each containing a representation of contralateral space, were identified anterior to VO-2. These two areas were found to be located within the posterior PHC extending along the collateral sulcus and flanked by the lingual gyrus and the posterior portion of the parahippocampal gyrus on one side and the medial fusiform gyrus on the other side. Following a naming convention based on approximate anatomical landmarks, we will refer to these two areas as parahippocampal cortical areas $\mathrm{PHC}-1$ and PHC-2. The posterior border of PHC-1 was formed by LVF angles (Figs. 1, 2, left panels, blue, dashed), forming the shared border with VO-2. The polar phase map of $\mathrm{PHC}-1$ progressed within posterior collateral sulcus from angles within the LVF to those within the UVF close to the UVM (red, dotted), constituting the shared border with PHC-2, with a systematic polar angle representation of the contralateral hemifield (Fig. $3 A, B$ ). A mirror reversal of the polar angle representation extending from the upper to the LVF was found in PHC-2 (Fig. $3 A, B$ ); the anterior border of PHC-2 was formed by LVF angles (Figs. 1, 2, left panels, blue, dashed). Additional examples are shown in supplemental Figures 3-5 (available at www.jneurosci.org as supplemental material). For PHC-1, hemifield representations of contralateral visual space were identified in all 16 hemispheres, as seen in the RH of subjects S1 and S2 (Fig. 1, left panels). Similar to the observations for hV4 and VO- $1 / 2$, the border shared by PHC- 1 and PHC-2 was often formed by a continuous representation of UVF angles, such as the one seen in the RH of subject S2 (Fig. 1, left panel, 13 of 16 hemispheres), but was sometimes formed by UVF angles intermixed with those near the HM, as seen in the LH of subject S2 (Fig. 2, left panel, 3 of 16 hemispheres). For PHC-2, all subjects showed a consistent pattern of phase angles within the UVF progressing anterior and medial to a representation of the HM, as seen in the LH of subjects S1 and S2 (Fig. 2, left panel). An additional progression to an LVF representation was found in PHC-2 for most subjects, as seen in the LH of subjects S1 and S2 (Fig. 2, left panel, 11 of 16 hemispheres).

We identified a progression of eccentricity within PHC-1/2, with the foveal representation located on the medial fusiform gyrus. This foveal representation was typically separated from the 
foveal representation of $\mathrm{VO}-1$ and $\mathrm{VO}-2$ (12 of 16 hemispheres, e.g., RHs of subjects S1 and S2) (Fig. 1, right panel, asterisk) but was found to be continuous with the fovea of VO- 1 and VO-2 in some cases (4 of 16 hemispheres). The foveal representation was typically located on the anterior/inferior border of PHC-1 and PHC-2 with the peripheral representations of PHC- 1 and PHC-2 bordering the presumed far peripheral representation of ventral V3 (not measured in the current experiment). Both areas exhibited a sudden transition from foveal to peripheral representations, which has been reported previously for other topographically organized higher-order visual areas (Larsson and Heeger, 2006; Swisher et al., 2007). Mean Talairach coordinates for left and right $\mathrm{PHC}-1$ were $-27,-54,-5$ and $+31,-52,-5$, respectively, and for PHC-2 were $-28,-46,-5$ and $+32,-44,-5$ (Table 1). The mean activation sizes of PHC-1 and PHC-2 were 1378 and 1642 $\mathrm{mm}^{3}$, which is 26 and $31 \%$ the size of $\mathrm{V} 1$, 54 and $64 \%$ the size of $\mathrm{hV} 4,120$ and $143 \%$ the size of VO-1, and 95 and $113 \%$ the size of VO-2 (Table 2, Fig. 4).

To evaluate the strength of the stimulus-evoked signal relative to noise in PHC-1 and PHC-2, the response amplitudes were plotted as a function of temporal frequency for the polar angle and eccentricity measurements (Fig. 5).

The temporal frequency histograms were derived for each subject and each hemisphere and then averaged across subjects to yield group data. For each subject, the response at the SF of six cycles was several SEs greater than the mean response across all other frequencies, demonstrating a strong link between the measured neural response and stimulus location. For the polar angle component, the average percentage signal changes at the SF for right and left PHC- 1 were $1.70 \pm 0.30$ and $1.53 \pm 0.35$ and for right and left PHC-2 were $0.33 \pm 0.06$ and $0.32 \pm 0.09$. For the eccentricity component, the average percentage signal changes at the SF for right and left PHC- 1 were $1.20 \pm 0.15$ and $1.0 \pm$ 0.2 and for right and left PHC-2 were $0.47 \pm 0.10$ and $0.30 \pm$ 0.06 . For both the polar angle and eccentricity components, the response at the SF ( 6 cycles) was significantly greater than noise $\left[\right.$ all $t_{(7)}$ values $>4.4$; $p$ values $<0.01$ ]

\section{Visual field representations of ventral visual areas}

The visual field representations for $\mathrm{V} 1, \mathrm{hV} 4, \mathrm{VO}-1, \mathrm{VO}-2$, PHC-1, and PHC-2 were computed by aligning the eccentricity and polar angle maps for each subject on the surface and extracting all surface nodes that had a significant phase value for both measurements. The location of each surface node with respect to eccentricity and polar angle was then plotted for each area and subject to yield an estimate of the visual field representation, as shown for the group of subjects and for each individual subject in Figure 6 . The inner sector represents the foveal $5^{\circ}$, the midsector eccentricities between 5 and $10^{\circ}$, and the outer sector eccentricities between 10 and $15^{\circ}$. Blue dots denote data from the $\mathrm{LH}$ and red dots those from the RH. All areas represented almost exclu- sively the contralateral visual field: $91 \pm 1 \%$ of nodes in area V1, $96 \pm 1 \%$ in hV4, $97 \pm 1 \%$ in $\mathrm{VO}-1,98 \pm 1 \%$ in $\mathrm{VO}-2,95 \pm 2 \%$ in PHC-1, and $87 \pm 4 \%$ in PHC-2 (contralateral vs ipsilateral visual field: all $t_{(7)}$ values $>8.5$; $p$ values $\left.<0.0001\right)$. Data were further evaluated for UVF versus LVF representations. There was a significantly greater number of nodes preferring the UVF over the LVF in hV4 (UVF, $65 \pm 4 \%$ ), VO-1 (UVF, $61 \pm 3 \%$ ), VO-2 (UVF, $72 \pm 4 \%$ ), and PHC-2 (UFV, $76 \pm 3 \%$; all $t_{(7)}$ values $>3.277$; $p$ values $<0.05$ ), with a nonsignificant trend in PHC-1 (UVF, $62 \pm 6 \% ; t_{(7)}=1.9 ; p=0.097$ ). In contrast, cortical area V1 did not show such an upper field bias (UVF, $48 \pm 2 \%$; $t_{(7)}=$ $-0.681 ; p>0.518)$. Consistent with the cortical magnification of foveal representations, area V1 showed a relatively larger representation of eccentricities up to $7.5^{\circ}\left(68 \pm 2 \%\right.$ of nodes; $t_{(7)}=$ 5.97; $p<0.001$ ). Areas hV4 and VO-1 also showed a strong bias toward foveal and parafoveal eccentricities up to $7.5^{\circ}(\mathrm{hV} 4,95 \pm$ $2 \%$ of nodes; VO-1, $86 \pm 4 \%$; both $t_{(7)}$ values $>5.141$; $p$ values $<0.001)$. PHC-1 and PHC-2 showed a significantly larger number of nodes preferring eccentricities ranging between $7.5^{\circ}$ and $15^{\circ}(83 \pm 2 \%$ of nodes in each area) compared with eccentricities up to $7.5^{\circ}$ (both $t_{(7)}$ values $>9.75$; $p$ values $<0.0001$ ), suggesting that these areas have large RFs, thereby making it difficult to attain reliable estimates of detailed eccentricity maps using a traveling wave paradigm (Larsson and Heeger, 2006; Wandell et al., 2007).

\section{Comparison of attentionotopy and retinotopic maps}

In a subset of subjects $(n=4)$, we performed an additional study that used standard retinotopic mapping with rotating wedge and 
Table 1. Talairach coordinates for right and left hemispheres of hV4, V0 1/2, PHC 1/2, and PPA

\begin{tabular}{|c|c|c|c|c|c|c|}
\hline & \multicolumn{3}{|l|}{ Left hemisphere } & \multicolumn{3}{|c|}{ Right hemisphere } \\
\hline & $x$ & $y$ & $z$ & $x$ & $y$ & $z$ \\
\hline \multicolumn{7}{|l|}{ hV4 } \\
\hline $\begin{array}{l}\text { Mean } \\
\text { Range }\end{array}$ & $\begin{array}{r}-23 \pm 3.5 \\
(-28,-17)\end{array}$ & $\begin{array}{c}-75 \pm 4.4 \\
(-80,-70)\end{array}$ & $\begin{array}{l}-10.8 \pm 6.2 \\
(-19,-1)\end{array}$ & $\begin{array}{l}25.5 \pm 5.8 \\
(16,35)\end{array}$ & $\begin{array}{l}-76.8 \pm 4.5 \\
(-83,-71)\end{array}$ & $\begin{array}{c}10.8 \pm 6 \\
(-25,-6)\end{array}$ \\
\hline \multicolumn{7}{|l|}{ v0-1 } \\
\hline $\begin{array}{l}\text { Mean } \\
\text { Range }\end{array}$ & $\begin{array}{l}-27.4 \pm 4.9 \\
(-35,-18)\end{array}$ & $\begin{array}{l}-68.6 \pm 6.2 \\
(-80,-60)\end{array}$ & $\begin{array}{l}-8 \pm 4.2 \\
(-16,-2)\end{array}$ & $\begin{array}{l}27.4 \pm 5.2 \\
(18,34)\end{array}$ & $\begin{array}{l}-66.8 \pm 6.2 \\
(-76,-57)\end{array}$ & $\begin{array}{l}-8.2 \pm 3.7 \\
(-15,-3)\end{array}$ \\
\hline \multicolumn{7}{|l|}{ v0-2 } \\
\hline $\begin{array}{l}\text { Mean } \\
\text { Range }\end{array}$ & $\begin{array}{l}-26.1 \pm 3.5 \\
(-32,-21)\end{array}$ & $\begin{array}{l}-59.6 \pm 5.8 \\
(-70,-54)\end{array}$ & $\begin{array}{l}-7 \pm 4.1 \\
(-11,2)\end{array}$ & $\begin{array}{l}25.4 \pm 4.5 \\
(19,31)\end{array}$ & $\begin{array}{l}-60.3 \pm 4.6 \\
(-69,-53)\end{array}$ & $\begin{array}{l}-7.4 \pm 4.6 \\
(-14,1)\end{array}$ \\
\hline \multicolumn{7}{|l|}{ PHC-1 } \\
\hline $\begin{array}{l}\text { Mean } \\
\text { Range }\end{array}$ & $\begin{array}{c}-27 \pm 4.1 \\
(-35,-22)\end{array}$ & $\begin{array}{l}-53.5 \pm 8.5 \\
(-70,-44)\end{array}$ & $\begin{array}{l}-5.3 \pm 3.1 \\
(-9,-1)\end{array}$ & $\begin{array}{l}30.6 \pm 4.4 \\
(22,37)\end{array}$ & $\begin{array}{l}-51.8 \pm 5.5 \\
(-61,-45)\end{array}$ & $\begin{array}{l}-5.3 \pm 3.4 \\
(0,-9)\end{array}$ \\
\hline \multicolumn{7}{|l|}{ PHC-2 } \\
\hline $\begin{array}{l}\text { Mean } \\
\text { Range }\end{array}$ & $\begin{array}{c}-28 \pm 5 \\
(-34,-17)\end{array}$ & $\begin{array}{l}-45.8 \pm 9.3 \\
(-64,-36)\end{array}$ & $\begin{array}{l}-4.9 \pm 1.7 \\
(-7,-2)\end{array}$ & $\begin{array}{l}32 \pm 2.6 \\
(28,36)\end{array}$ & $\begin{array}{c}-44 \pm 4.6 \\
(-51,-36)\end{array}$ & $\begin{array}{l}-4.9 \pm 3.8 \\
(1,-9)\end{array}$ \\
\hline \multicolumn{7}{|l|}{ PPA } \\
\hline $\begin{array}{l}\text { Mean } \\
\text { Range }\end{array}$ & $\begin{array}{c}-25 \pm 5 \\
(-30,-20)\end{array}$ & $\begin{array}{r}-48 \pm 6.5 \\
(-59,-39)\end{array}$ & $\begin{array}{l}-7.4 \pm 4.2 \\
(-14,-2)\end{array}$ & $\begin{array}{c}28 \pm 5 \\
(21,37)\end{array}$ & $\begin{array}{c}-43 \pm 4.9 \\
(-49,-34)\end{array}$ & $\begin{array}{l}-8.5 \pm 3.9 \\
(-13,-3)\end{array}$ \\
\hline
\end{tabular}

Table 2. Surface volume estimates (measured between pial and white matter) for right and left hemispheres of hV4, V0-1/2, and PHC-1/2

\begin{tabular}{ccccccc}
\hline Hemisphere & V1 & hV4 & V0-1 & V0-2 & PHC-1 & PHC-2 \\
\hline Surface volume & & & & & & \\
Right & 2812.5 & 1410.4 & 627.9 & 716.2 & 723.9 & 911.9 \\
SD & 568.6 & 328.8 & 240.6 & 143.5 & 208 & 182.7 \\
\% of V1 & 100 & 50 & 22 & 25 & 26 & 32 \\
Left & 2504.4 & 1152.1 & 517.2 & 736 & 654.3 & 730 \\
SD & 526.6 & 288.9 & 162.7 & 228.5 & 189.7 & 182.4 \\
\% of V1 & 100 & 46 & 21 & 29 & 26 & 29 \\
Both & 5317 & 2562.5 & 1145.1 & 1452.2 & 1378.3 & 1641.9 \\
SD & 1015 & 527 & 337.1 & 314 & 353.9 & 350 \\
\% of V1 & 100 & 48 & 22 & 27 & 26 & 31 \\
\hline
\end{tabular}

expanding or contracting ring stimuli that were presented while subjects performed a luminance detection task at fixation. The same amount of data as in the attentionotopy studies were collected in these subjects. The data obtained in the retinotopic mapping studies were then qualitatively and quantitatively compared in the four subjects to address two major issues. First, we asked whether directed attention to the mapping stimuli was a requirement to reveal orderly maps in anterior parts of ventral visual cortex, because this part of cortex may not activate well under passive viewing conditions. Second, we were concerned that the visual field representation yielded with the attentionotopy paradigm may be distorted as a result of the attentional manipulation. As is evident from the retinotopic maps in the right hemisphere of subject S3 (Fig. 7A), qualitatively similar visual field maps were identified in ventral visual cortex using standard retinotopic mapping techniques. Importantly, the area borders identified with standard retinotopy matched the borders identified with attentionotopy. Also, individual variations of visual area representations remained consistent between paradigms within a given subject (Fig. 7A) (for additional examples, see supplemental Fig. 6, available at www.jneurosci.org as supplemental material). For example, discontinuous representations of the border between VO-2 and PHC-1 were identified in two of the eight hemispheres in the attentionotopy studies, which were also present in the same subjects and hemispheres in the retinotopy studies. Furthermore, the foveal representation of PHC-1 and PHC-2 could be clearly identified in all subjects with both mapping approaches with the same typical separation from the

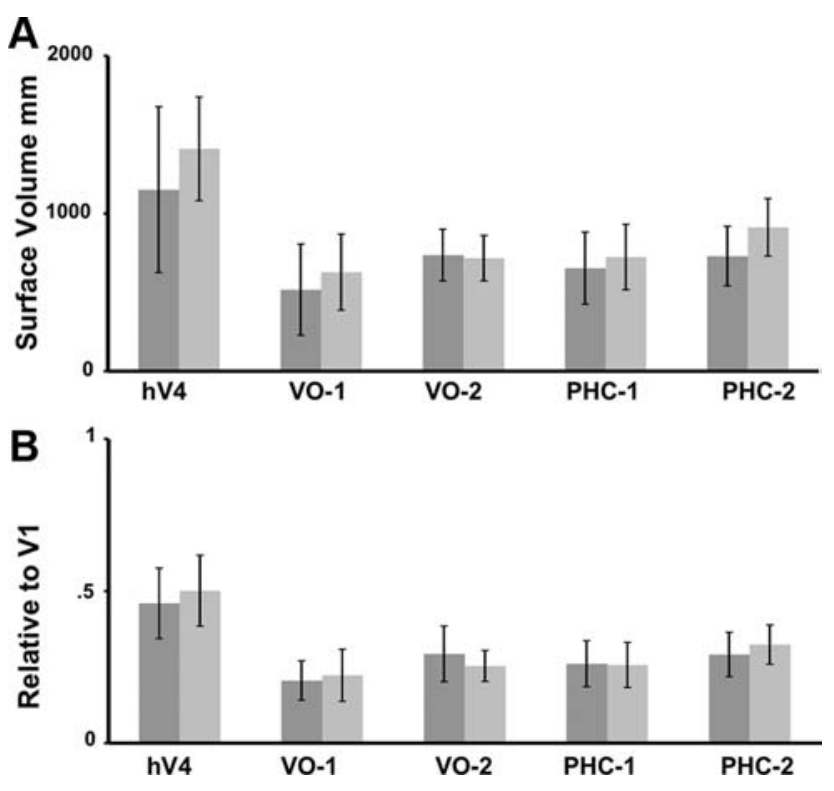

Figure 4. Estimated surface volume for V1, hV4, V0-1, V0-2, PHC-1, and PHC-2. A, Surface volumes in cubic millimeter for right (light gray) and left (dark gray) hemispheres of V1, hV4, V0-1, V0-2, PHC-1, and PHC-2 ( $n=16)$. B, Surface volumes for hV4, V0-1, V0-2, PHC-1, and PHC-2 for RH and LH calculated as a percentage of $\mathrm{V} 1$ (same data as in $\boldsymbol{A}$ ). Vertical bars indicate SEM. On average, hV4 was approximately half the size of V1, and visual areas V0-1 to PHC-2 were between one-quarter and one-third the size of V1.

foveal representation of VO-1/2 (Fig. 7A). Although there were significantly fewer nodes activated in the retinotopic mapping study in PHC-1 and PHC-2 (ranging from 14 to $17 \%$ fewer nodes on average, $\left.t_{(3)}>5.7 ; p<0.05\right)$, indicating a main effect of attention in terms of response enhancement, the relative representations of the visual field for both areas were almost identical between the two paradigms (Fig. 7C). All four subjects had dominant representations of the contralateral visual field in PHC-1 and PHC-2 for both retinotopy and attentionotopy datasets (contralateral vs ipsilateral; all $t_{(3)}$ values $>4.77$; $p$ values $<0.05$ ) (Fig. $7 B$ ) with $89 \pm 6 \%$ compared with $93 \pm 4 \%$ in PHC-1 in the attentionotopy and retinotopy studies, respectively, and with $78 \pm 5 \%$ compared with $78 \pm 6 \%$ in PHC-2 (both $t_{(3)}$ values $<1.4$; $p$ values $>0.05$ ). All four subjects had significantly greater 


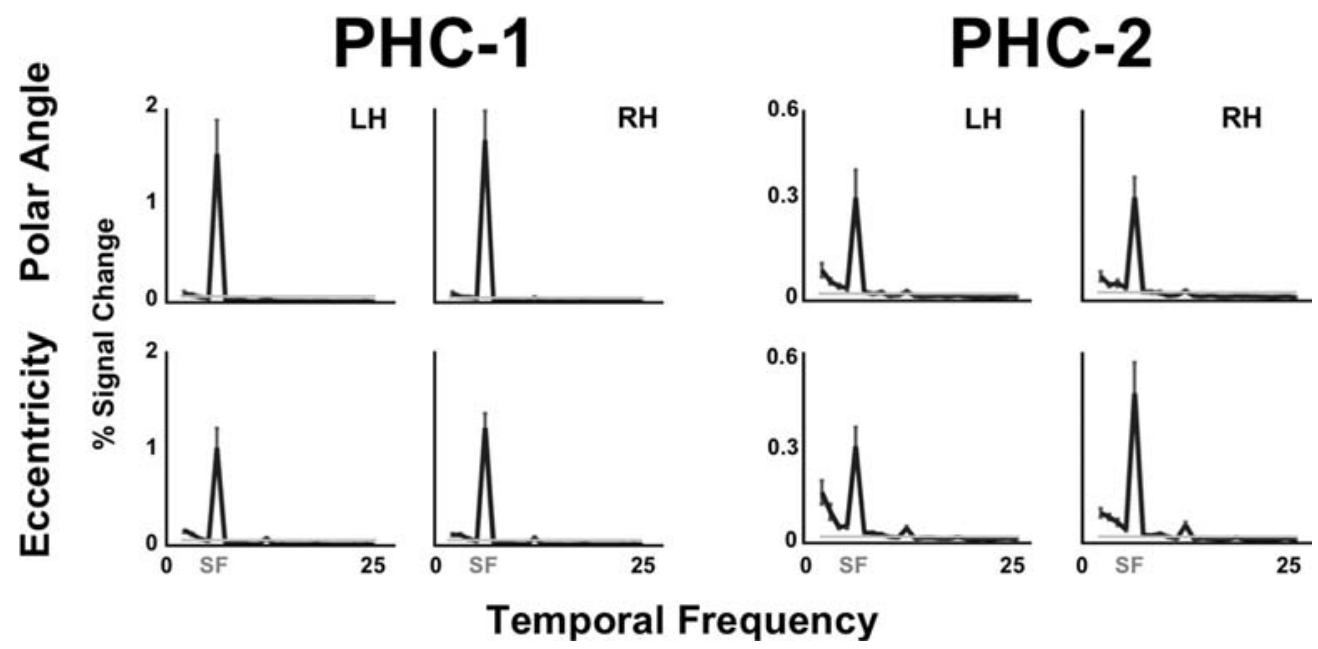

Figure 5. Response amplitudes as a function of temporal frequency in PHC-1 and PHC-2. Results from attentionotopy studies. Data were averaged across 16 hemispheres for PHC -1 and PHC-2. The top panel shows the results for the polar angle measurements, the bottom those for the eccentricity measurements. The response at the SF was significantly greater than the response at other frequencies. Light gray bars denote the noise level, calculated as the mean amplitude across all frequencies.

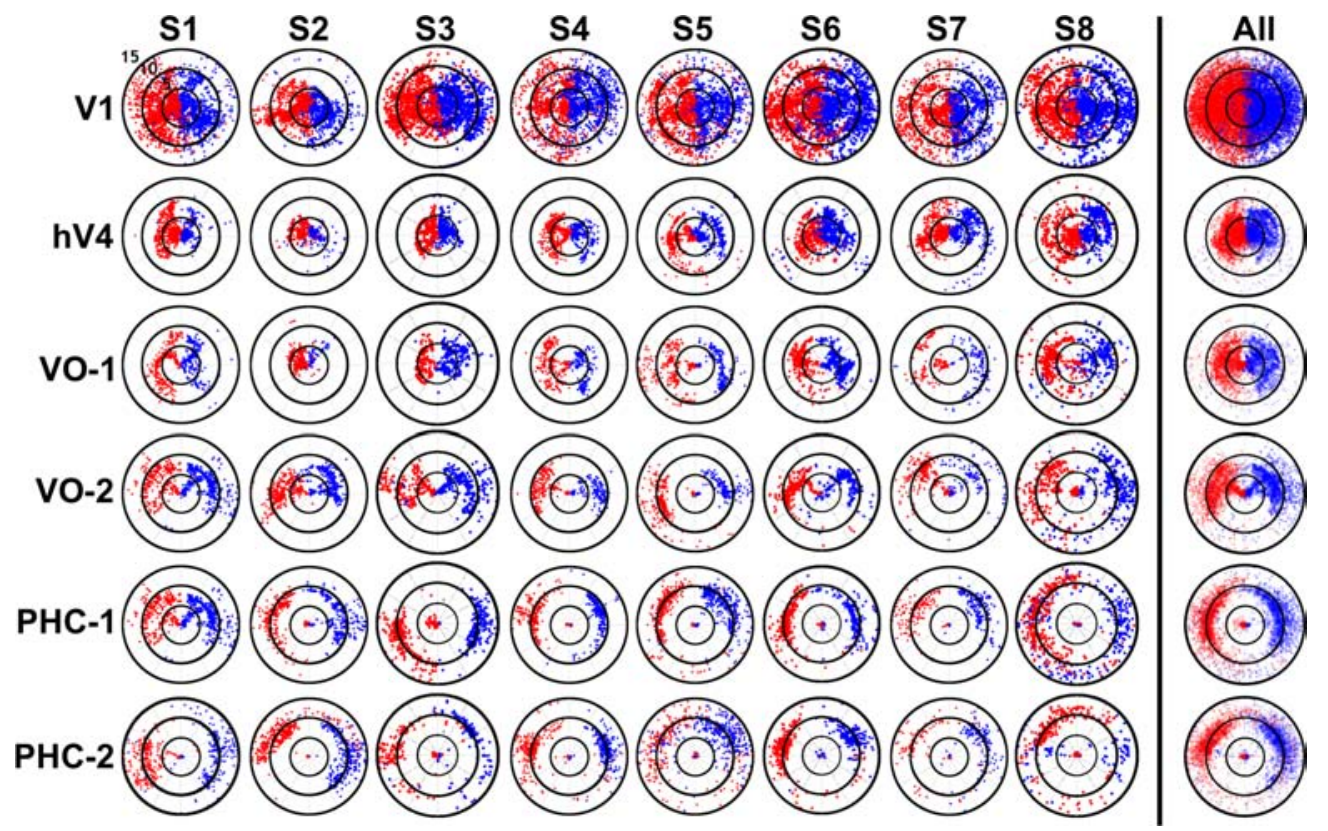

Figure 6. Visual field representation in areas V1, hV4, V0-1, V0-2, PHC-1, and PHC-2. Vertice plots from each individual subject and group analysis $(n=8)$ based on polar and eccentricity maps thresholded at $1.5 \mathrm{~s}$ of the cycle SEM variance (see Materials and Methods) obtained in the attentionotopy studies. Surface nodes that had significant phase estimates for both polar angle and eccentricity were plotted such that each point represents the corresponding preferred visual field location for a given node. Red and blue points indicate data from the RH and LH, respectively. All areas showed strong contralateral preference. HV4, V0-1, V0-2, PHC-2, and to some degree PHC-1 showed a smaller representation of the LVF relative to the UVF. HV4 and V0-1 demonstrated an almost exclusive activation of the visual field representation within $0-7.5^{\circ}$ eccentricity. In contrast, PHC -1 and PHC -2 represented the fovea and eccentricities ranging from 7.5 to $15^{\circ}$ better than other eccentricities.

peripheral representations between 7.5 and $15^{\circ}$ in PHC-1 and PHC-2 for both retinotopy and attentionotopy datasets $\left(7.5-15^{\circ}\right.$ vs $0-7.5^{\circ}$; all $t_{(3)}$ values $=8.7 ; p$ values $\left.<0.05\right)($ Fig. $7 C)$. The peripheral representations of the retinotopy data did not significantly differ from the attentionotopy data in PHC-1 with $85 \pm 2 \%$ compared with $83 \pm 3 \%$, nor in PHC-2 with $77 \pm 8 \%$ compared with $78 \pm 4 \%$ (both $t_{(3)}$ values $<0.51$; $p$ values $>0.05$ ). All four subjects also had a significantly greater UVF representations in PHC-2 for both retinotopy and attentionotopy datasets (UVF vs LVF; both $t_{(3)}$ values $>5.19$; $p$ values $<0.05$ ) (Fig. $7 B$ ) with $78 \pm$ $4 \%$ compared with $74 \pm 9 \%$ for the attentionotopy and retinotopy experiments, respectively $\left(t_{(3)}=0.27 ; p>0.05\right)$. Together, these results suggest, first, that that the visual field maps in the posterior PHC can be activated under passive viewing conditions using standard retinotopy techniques and, second, that the visual field representations within the $\mathrm{PHC}$ areas were not distorted as a result of the allocation of spatial attention to the mapping stimuli.

To further evaluate the similarity of phase estimates between the attentionotopy and retinotopy data on a node-by-node basis, AIs were calculated for both hemispheres in each of the four subjects (see Materials and Methods) (Fig. 7B) (supplemental Fig. 6, available at www.jneurosci.org as supplemental material). The AI values range from 1 , which indicates perfect phase alignment between retinotopy and attentionotopy, to 0 , which indi- 
A

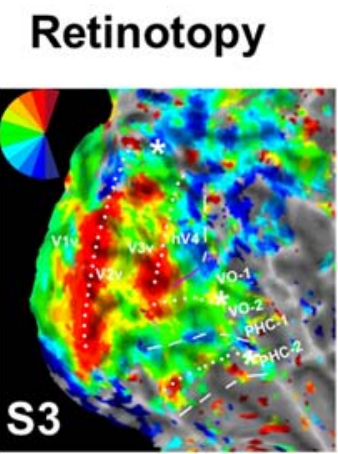

Attentionotopy
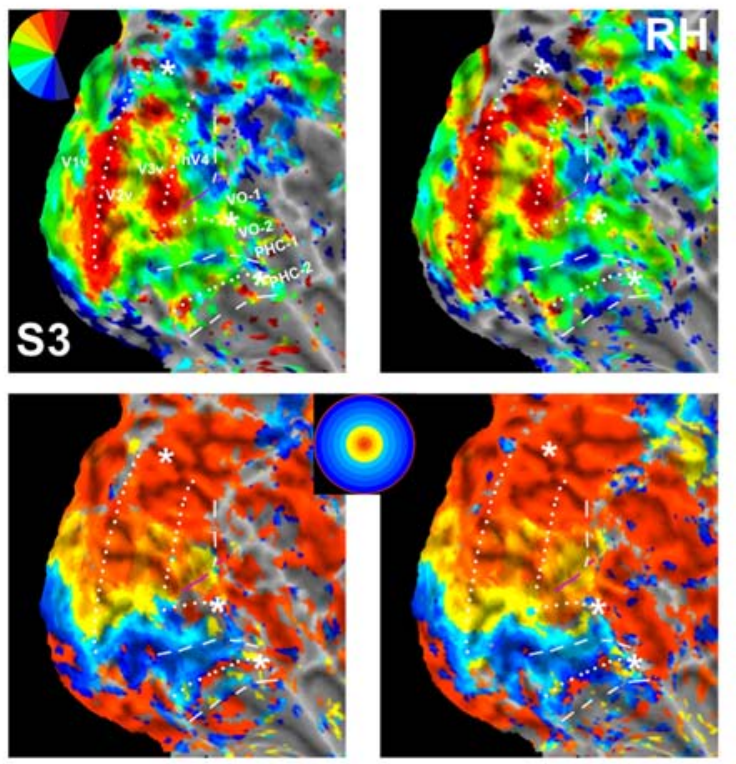

B

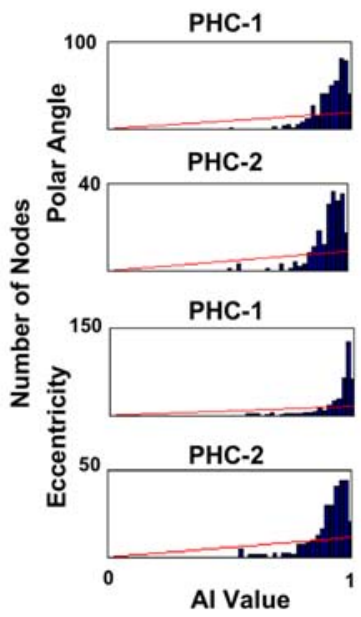

\section{Retinotopy}

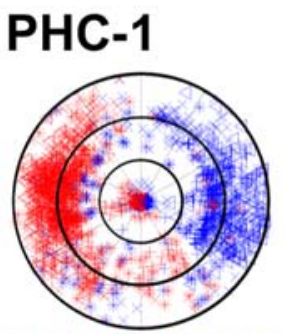

$\mathrm{N}=1415 \quad \mathrm{~N}=1125$
PHC-2

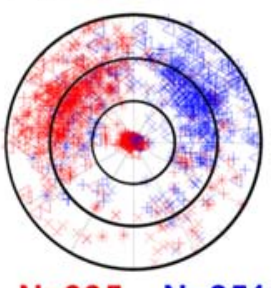

$\mathrm{N}=995 \quad \mathrm{~N}=851$

Attentionotopy
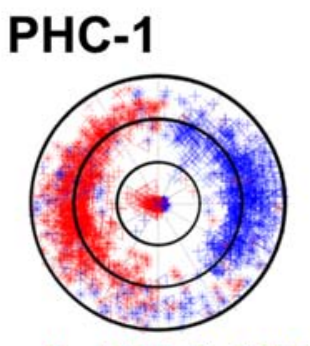

$\mathrm{N}=1637 \quad \mathrm{~N}=1358$

\section{PHC-2}

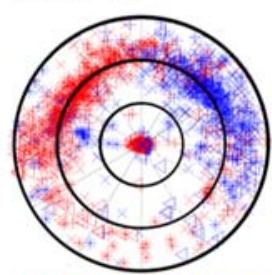

$\mathrm{N}=1175 \quad \mathrm{~N}=998$

Figure 7. Comparison of maps obtained in retinotopy and attentionotopy studies. $\boldsymbol{A}$, Polar angle and eccentricity maps of ventral visual cortex from subject $\$ 3$ (left hemisphere). The left shows the maps obtained with standard retinotopy mapping procedures with subjects performing a fixation task. The right shows the results for the attentionotopy studies from the same subject. The overall activation pattern is similar and consistent across the two mapping approaches. The posterior and anterior borders of PHC-1 were apparent in the retinotopy studies, albeit a somewhat more patchy representation. Particularly, the anterior border of PHC-2 was more clearly seen in the attentionotopy data in this subject. The eccentricity maps look nearly identical with the different measurements. All maps were thresholded at $1.5 \mathrm{~s}$ of the cycle SEM variance. $\boldsymbol{B}$, Histograms of alignment indices (AI $=1-|\Delta \phi| / \pi)$ for PHC 1 and PHC-2 for subject S3. The index values peak around an index value close to 1 , which indicates good alignment between attentionotopy phase estimates and retinotopy estimates for polar angle and eccentricity. The red line illustrates a distribution for uncorrelated data. $C$, Vertice plots for PHC- 1 and PHC-2 from retinotopy and attentionotopy studies $(n=4)$. A smaller amount of nodes was activated in PHC- 1 and PHC -2 in the retinotopy compared with the attentionotopy studies. However, the overall characteristics of visual field representation are consistent across the two paradigms, particularly the greater representation of the UVF in PHC-2 and the emphasis on peripheral eccentricities. For other conventions and additional details, see Figure 5.

cates that the values obtained in the two datasets were completely out of phase by $180^{\circ}$. Histograms of AIs are shown for the PHC areas from subject S3 in Figure $7 B$ (also see supplemental Fig. 6, available at www.jneurosci.org as supplemental material). The mean index values for the RH of PHC-1 and PHC-2 were $0.92 \pm$ 6 and $0.92 \pm 8$, illustrating the strong alignment between attentionotopy and retinotopy measurements in this subject (Fig. 7A). Mean AI values were averaged across subjects to yield group data. For polar angle phase estimates, the group AI was $0.96 \pm 0.01$ in the RH and $\mathrm{LH}$ for VO- $1,0.94 \pm 0.01$ in the RH and $0.95 \pm 0.01$ in the $\mathrm{LH}$ for VO-2, $0.93 \pm 0.01$ in the $\mathrm{RH}$ and $0.91 \pm 0.02$ in the LH for PHC-1, and $0.91 \pm 0.01$ in the RH and $0.91 \pm 0.01$ in the LH for PHC-2. For eccentricity phase estimates, the group AI was $0.90 \pm 0.01$ in the $\mathrm{RH}$ and $0.88 \pm 0.02$ in the $\mathrm{LH}$ for $\mathrm{VO}-1,0.86 \pm$ 0.01 in the RH and $0.86 \pm 0.01$ in the $\mathrm{LH}$ for VO-2, $0.90 \pm 0.01$ in the RH and $0.89 \pm 0.01$ in the LH for PHC- 1 , and $0.88 \pm 0.01$ in the $\mathrm{RH}$ and $0.88 \pm 0.02$ in the $\mathrm{LH}$ for PHC-2. AI values were significantly above chance (see Materials and Methods) (polar angle, all $t_{(3)}$ values $>16.62$, $p$ values $<0.001$; eccentricity, all $t_{(3)}$ values $>19.10, p$ values $\left.<0.001\right)$. Additionally, the calculated correlation coefficients of each ROI were highly significant for each subject's polar angle and eccentricity measurements (all $r$ values $>0.33, p$ values $<10^{-10}$; median polar, $r=0.60, p<$ $10^{-15}$; median eccentricity, $r=0.83, p<10^{-15}$ ) (see Materials and Methods), demonstrating that there was good alignment throughout each ROI for every subject. These analyses indicate that both paradigms yielded highly consistent results for both polar angle and eccentricity measurements for individual subjects.

Reproducibility of attentionotopy maps

In an additional experiment, we established the reliability of the polar angle and eccentricity measurements within ventral visual cortex by rescanning two subjects using an identical experimental paradigm (i.e., the attentionotopy study design). The resulting 
maps were highly reproducible within subjects, as indicated qualitatively by the similarities in characteristics of polar phase and eccentricity progressions discussed above and quantitatively by the virtually identical visual field representations in PHC-1 and PHC-2 (supplemental Fig. 7, available at www.jneurosci.org as supplemental material). AI indices were calculated between the two attentionotopy experiments for both polar angle and eccentricity phase estimates (supplemental Fig. 7, right column, available at www.jneurosci.org as supplemental material). Strong correlations ranging between 0.89 and 0.95 were found within $\mathrm{PHC}$ areas for each subject. For comparison, AI values for V1 ranged between 0.94 and 0.96 for these subjects. Together with the retinotopy experiments, these studies provide important test-retest verification for the newly described retinotopic maps in PHC-1 and PHC-2.

\section{Responses to object stimuli}

Because ventral visual cortex is known to be activated by object stimuli (Malach et al., 1995; Kanwisher et al., 1997; Epstein and Kanwisher, 1998), we probed the response properties of ventral visual areas to various object categories. The retinotopic areas described in detail above were used as ROIs to examine the response properties evoked by scenes, faces, inanimate objects, and scrambled images. Percentage signal changes of functional MRI (fMRI) signals were calculated for each object category in areas hV4, VO-1, VO-2, PHC-1, and PHC-2 (Fig. $8 \mathrm{~B}$ ). In all areas, there was a main effect of object category (all $F_{(3,21)}$ values $=4.83 ; p$ values $\left.<0.01\right)$. Pairwise comparisons revealed significantly stronger responses evoked by scene stimuli than by face stimuli in all areas (all $t_{(7)}$ values $>2.51$; $p$ values $<0.05$ ). However, neural responses in areas hV4 and VO-1 did not discriminate between scene, object, or scrambled stimuli (all $t_{(7)}$ values $<2.14$; $p$ values $>0.05$ ) (Fig. $8 B$ ). Scene stimuli exhibited significantly stronger responses than all other categories (inanimate object, scrambled, and face stimuli) in VO-2, PHC-1, and PHC-2 $\left(\right.$ all $t_{(7)}$ values $>3.66$; $p$ values $\left.<0.05\right)$ (Fig. $8 \mathrm{~B}$ ).

To examine neural responses in different parts of the visual field as well as the specificity of the responses within retinotopically organized ventral visual areas, 15 ROIs within hV4, VO-1, VO-2, PHC-1, and PHC-2 as well as adjacent (and lateral) to each area were sampled (Fig. 9A). Each ROI represented a $3 \mathrm{~mm}$ radius disc on the surface that was placed on the horizontal meridian of either foveal (denoted by red circles) or peripheral (denoted by blue circles) representations. In addition, a control ROI adjacent, but lateral to each foveal ROI, was defined (denoted by yellow circles). Percentage signal changes of fMRI signals evoked by scenes, faces, inanimate objects, and scrambled pictures were calculated for each of these ROIs. As shown in Figure 9B, a main effect of object category was observed in all foveal and peripheral 0.05 , uncorrected).
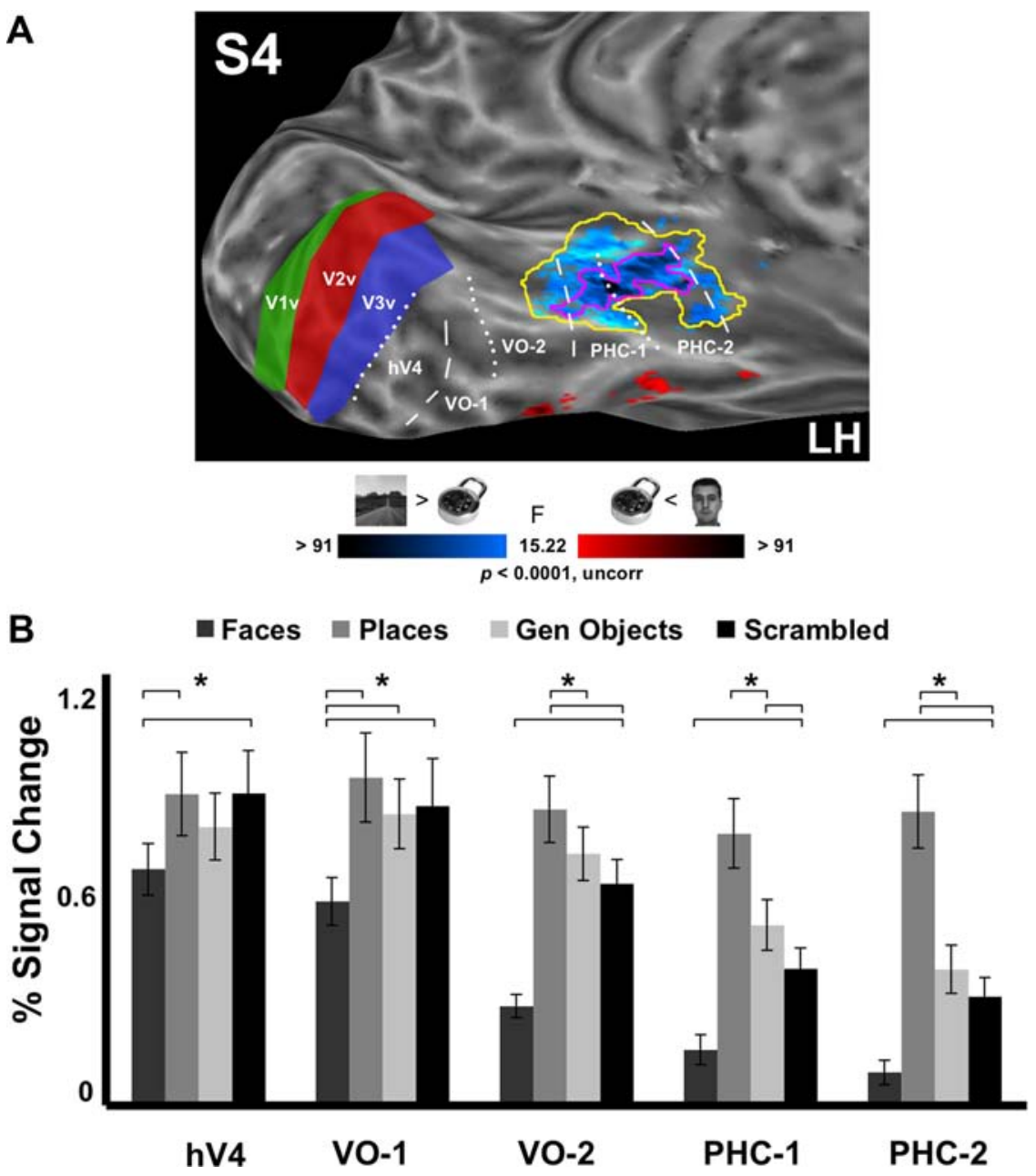

Figure 8. Responses to object stimuli in ventral visual cortex. A, Overlap of the PPA, as defined based on the contrast scenes Face-selective activations, as defined by contrasting faces and objects, are shown for additional reference. Outlines of the PPA defined at $p<0.001$ (yellow) and $p<10^{-10}$ (magenta) are shown. $\boldsymbol{B}$, FMRI signals in mean percentage signal change evoked by to scenes than to other object categories. Vertical bars denote significant differences between categories for paired $t$ tests ( $p<$

ROIs (all $F_{(3,21)}$ values $=5.40 ; p$ values $<0.01$ ), except for the foveal representation of hV4, which was similarly activated by all object categories $\left(F_{(3,21)}=1.19 ; p>0.05\right)$. Pairwise comparisons were computed for all foveal and peripheral ROIs (except for the fovea of hV4). Scene stimuli evoked significantly stronger responses than all other stimulus categories in the foveal ROIs of PHC-1 and PHC-2 and in the peripheral ROIs of VO-2, PHC-1, and PHC-2 (scene vs each other individual category per ROI; all $t_{(7)}$ values $>4.69$; $p$ values $<0.05$ ) (Fig. 9B). The remaining ROIs (foveal VO-1 and VO-2, peripheral hV4 and VO-1) responded more strongly to place, object, and scrambled pictures than to face stimuli (all $t_{(7)}$ values $>3.14$; $p$ values $<0.05$ ) but did not discriminate between the three former categories (all $t_{(7)}$ values $<1.95$; $p$ values $>0.05$ ) (Fig. $9 B$ ), except for the peripheral representation of $\mathrm{VO}-1$ that responded more strongly to places than to scrambled images $\left(t_{(7)}=2.78 ; p<0.05\right.$ ) (Fig. 9B). There were no significant differences in responses evoked by stimuli of any category in the ROIs adjacent to the defined topographic regions (all $F_{(3,21)}$ values $<2.54$; $p$ values $>0.05$ ) (Fig. 9B), except for the ROI lateral to VO-2 $\left(F_{(3,21)}=3.04 ; p<0.05\right)$, which showed significantly stronger responses for faces than scrambled pic- 
A

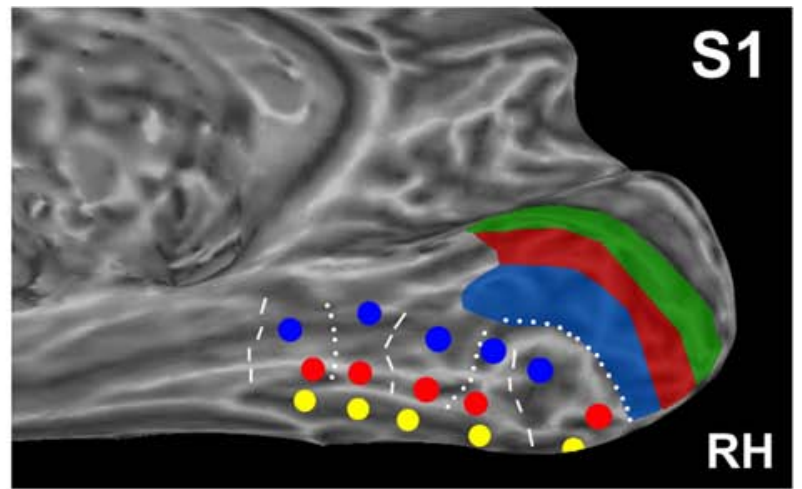

B

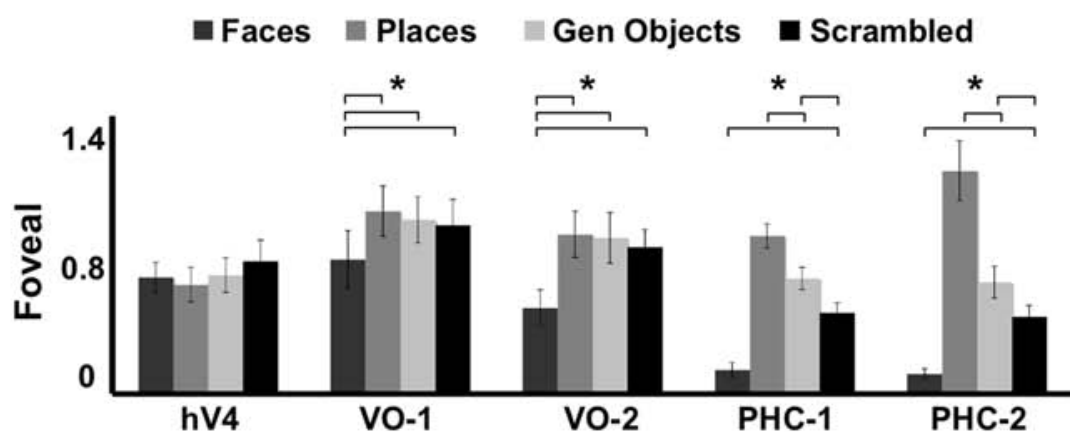

일

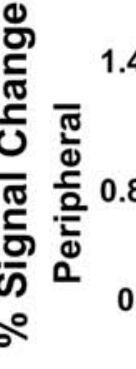

hV4

Vo-1

VO-2

PHC-1

PHC-2

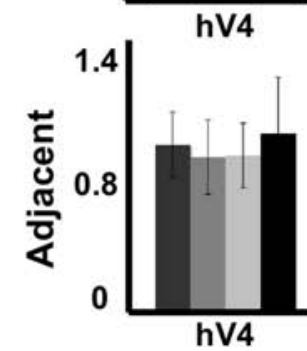

VO-1

VO-

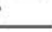
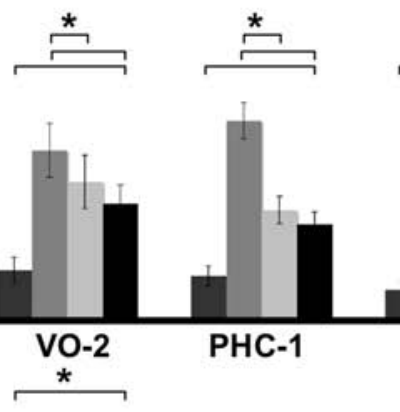

PHC-2

hV4

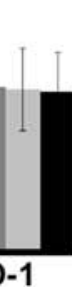

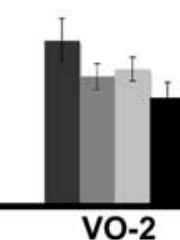

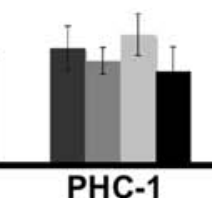

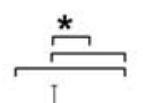

Figure 9. Responses to object stimuli in foveal and peripheral representations of hV4, V0-1, V0-2, PHC-1, and PHC-2. A, Locations of foveal, peripheral, and adjacent ROIs in relation to the borders of hV4, V0-1, V0-2, PHC-1, and PHC-2. B, FMRI signals in mean percentage signal change within foveal, peripheral, and adjacent $\mathrm{RO}$ Is of ventral visual areas evoked by various categories of stimuli. Data were averaged across hemispheres and subjects. PHC-1 and PHC-2 showed significantly greater responses to scenes than to other categories in both foveal and peripheral ROls. There were no significant differences in mean percentage signal change across categories for adjacent ROIs. Vertical bars indicate SEM. Horizontal bars denote significant differences between categories for paired $t$ tests $(p<0.05$, uncorrected).

tures $\left(t_{(7)}=2.55 ; p<0.05\right)$ (Fig. $\left.9 B\right)$, demonstrating the specificity of the response profiles within retinotopic areas. Together, these results suggest that PHC-1 and PHC-2 respond more strongly to places and scenes than to any other of the object categories tested here in both their peripheral and foveal representations.

To further quantify the object category response profiles of each area and to compare preferred responses for scenes across areas, a scene preference index was calculated that evaluates preferential responses for scenes relative to inanimate objects (SPI) (Fig. 10). This analysis confirmed the strong scene preference of PHC- 1 and PHC-2. For the SPI, the foveal ROIs of PHC- 1 and PHC-2 and the peripheral ROIs of VO-1, VO-2, PHC-1, and

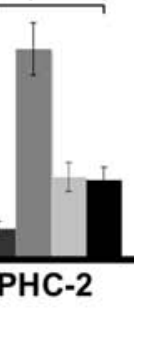

PHC-2 showed significant preference for scenes $\left(\right.$ all $t_{(7)}$ values $>2.54 ; p$ values $<0.05$ ) (Fig. 10). hV4, foveal VO-1, foveal $\mathrm{VO}-2$, and the ROIs adjacent and lateral to PHC-1 and PHC-2 showed no such preference. Scene preference increased from more posterior to anterior areas. Both PHC-1 and PHC-2 showed significantly stronger scene preference than VO-1 and VO-2 for both foveal and peripheral ROIs (all $t_{(7)}$ values $>5.3$; $p$ values $<0.001$ ), and PHC-2 showed significantly stronger scene preference than PHC-1 in both foveal and peripheral ROIs (both $t_{(7)}$ values $>3.686$; $p$ values $<0.01$ ).

Overlap of PHC areas with functionally defined PPA

Given that the foveal and peripheral representations of PHC-1 and PHC-2, as well as the areas as a whole, preferentially responded to pictures of scenes, we compared the location and extent of the functionally defined PPA (Epstein et al., 1999 ) in relation to our newly defined retinotopic areas. The PPA was defined as a contiguous cluster of activation within the collateral sulcus that responded more strongly to scenes than inanimate objects (thresholded at $p<0.0001$, uncorrected). Outlines of the extent of PPA in individual subjects as well as those of face-selective activations (faces $>$ object, thresholded at $p<0.0001$, uncorrected) are illustrated in supplemental Figures 3-5 (right panel; available at www.jneurosci.org as supplemental material). In all subjects, the PPA overlapped heavily with both PHC-1 and PHC-2 (Fig. 8A). The percentage of overlap was calculated for each hemisphere and then averaged across hemispheres to yield group data. At a statistical threshold of $p<0.0001,70.9 \pm 3 \%$ of the PPA overlapped with PHC-1 and PHC-2 in the RH and $67 \pm 7 \%$ in the LH. In 12 hemispheres of six subjects, a small portion of the PPA, $10.4 \pm 2 \%$ in the $\mathrm{RH}$ and $15.6 \pm 2 \%$ in the $\mathrm{LH}$, extended into the peripheral representation of VO-2. In 10 of 16 hemispheres, a small portion of the PPA, $18.8 \pm 3 \%$, extended farther anterior from PHC-2 into parahippocampal cortex. Conversely, $53.8 \pm$ $5 \%$ of PHC- 1 and PHC-2 overlapped with the PPA in the RH and $47.7 \pm 8 \%$ in the LH. The extent of the PPA activation, and therefore the percentage overlap between PHC and PPA, was greatly affected by the statistical significance at which the activation maps were thresholded. When adjusting the statistical threshold, the overlap of PPA with PHC for the RH and LH spanned from $63 \pm 4$ and $56 \pm 5 \%$ at a more lenient threshold of $p<0.001$ to $79 \pm 4$ and $76 \pm 6 \%$ at a more stringent threshold of $p<10^{-10}$, as illustrated in Figure 8 A. The overlap of PPA with PHC significantly differed between the three thresholds for both hemispheres (both $F_{(2,14)}$ values $>11.53$; $p$ values $<0.05$ ). 


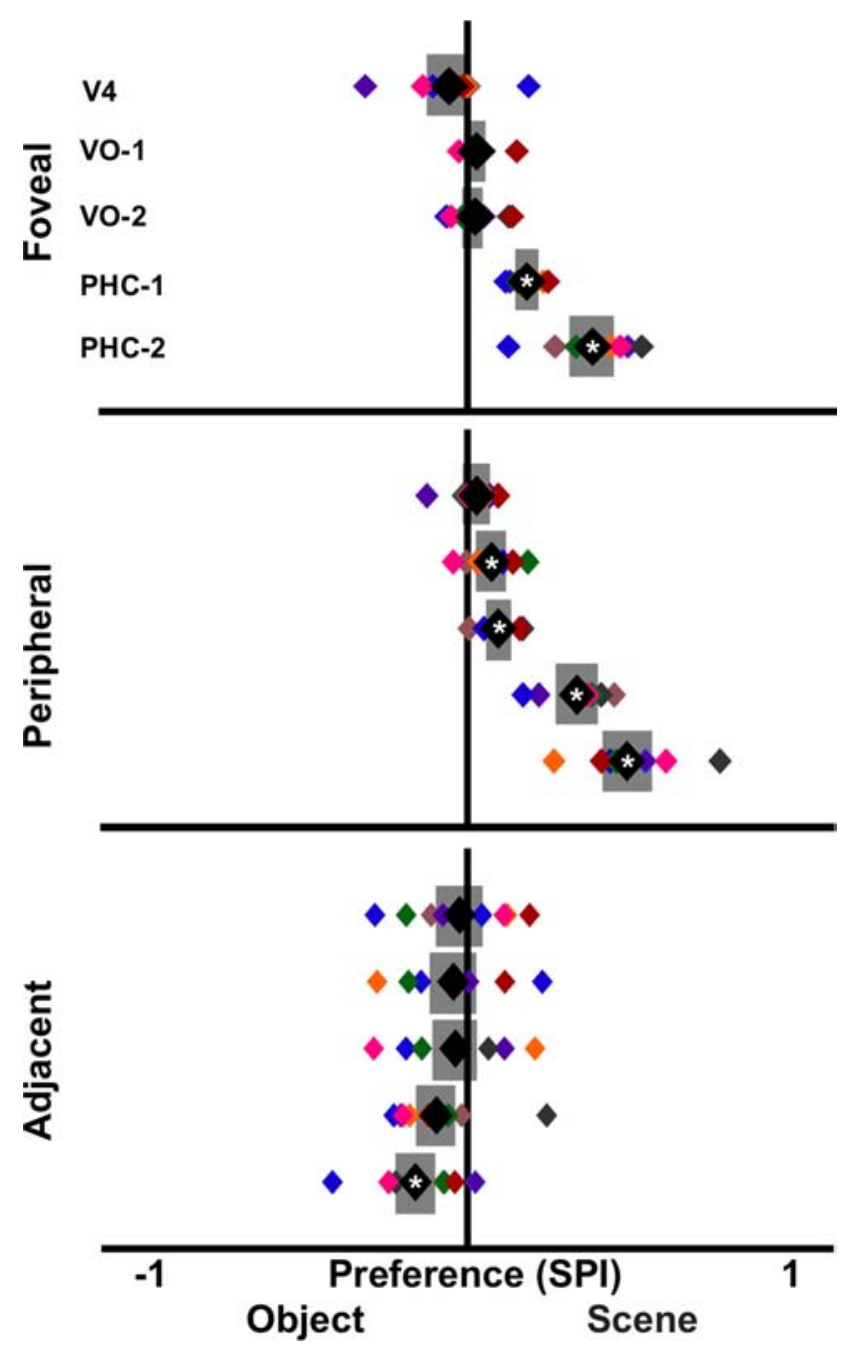

Figure 10. Scene preference index across ventral visual cortex. Index values were calculated by subtracting the mean percentage signal change obtained in response to the preferred category from those obtained in response to the general object category and dividing by the sum of the two. The index values range between 1 and -1 , with positive values showing preferred category selectivity for scenes and negative values showing preferred preference for the general object category. For each subject, data were averaged across hemispheres (colored diamonds) and averaged across all eight subjects (black diamond), with the shaded bar representing the SE. Both the foveal and peripheral ROIs in PHC-1 and PHC-2 showed strong preference for scenes. There was no significant preference for scenes in the ROls adjacent to PHC-1 and PHC-2.

Both hemispheres showed significant linear trends with percentage overlap between PPA and PHC increasing at more stringent thresholds (both $F_{(1,7)}$ values $>25.26$; $p$ values $<0.05$ ). At more liberal thresholds, the extent of the PPA activation spread farther posterior to PHC within VO-2 as well as anterior to PHC and beyond retinotopically defined cortex. However, the peak activations of all subjects fell within the PHC areas, and the average group Talairach coordinates for the peak activation of the PPA fell within PHC-2 for the $\mathrm{RH}$ and near the border of PHC-1 and PHC-2 for the LH (Table 1).

\section{Discussion}

We investigated the topographic organization of human ventral visual cortex using attentional wedge and ring tracking paradigms as well as standard phase-encoded retinotopic mapping in combination with high-resolution fMRI. Two previously not described retinotopic areas were identified within posterior PHC and anterior to the VO cluster, referred to as PHC-1 and PHC-2.
In each subject, both PHC areas exhibited blood oxygenation level-dependent modulations specifically in-phase with polar angle and eccentricity stimuli in both hemispheres. Cortex that lacks spatially specific representations would not show such specific phase-dependent modulations. When defining the borders of PHC, polar angle and eccentricity phase estimates were considered together. These areas shared a fovea and represented predominantly the contralateral visual field with a systematic progression of polar angle from the LVF to UVF in PHC-1 that was mirror reversed in PHC-2. Both areas showed a strong bias toward peripheral visual field locations, indicative of large RFs and similar to observations in other higher-order topographic areas (Larsson and Heeger, 2006; Swisher et al., 2007). The topographic organization within ventral visual cortex and the presented framework for identifying borders was consistent across all 16 hemispheres, despite some individual variability, as has been noted previously in higher-order cortex of both human and macaque (Gattass et al., 1988; Boussaoud et al., 1991; Brewer et al., 2005; Larsson and Heeger, 2006; Kastner et al., 2007; Konen and Kastner, 2008a).

In addition to identifying two new retinotopic areas in human ventral visual cortex, we also confirmed the topographic organization of areas hV4, VO-1, and $\mathrm{VO}-2$, as previously described by Wandell and colleagues (Wade et al., 2002; Brewer et al., 2005) (see also Tyler et al., 2005). Consistent with previous reports of $\mathrm{hV} 4$, we found that, although this area represented predominantly the contralateral hemifield in each hemisphere, coverage was particularly sparse at mid to far eccentricities for the LVF (Larsson and Heeger, 2006; Hansen et al., 2007). To account for this asymmetry in the UVF and LVF representations of V4, Hansen et al. (2007) have proposed an alternative mapping scheme for this region. They propose a region labeled dorsal V4, located adjacent to parafoveal dorsal V3 and representing the "missing" part of the LVF, an interpretation that has been controversial (for discussion, see Wandell et al., 2007). Our data cannot speak to this debate, because the coverage of the acquisition volume within dorsal visual cortex was limited as a result of the high-resolution $\mathrm{PMRI}$ protocol that we used. Two areas were located anterior to and contiguous with hV4, VO-1, and VO-2, which form together the VO cluster. Consistent with previous studies (Brewer et al., 2005; Wandell et al., 2005), we found that both VO areas represented predominantly the contralateral visual field with a preference for foveal and parafoveal eccentricities. The VO areas also exhibited a preference for the UVF, consistent with a UVF "hypertrophy" that has been observed previously within this part of cortex (Tyler et al. 2005).

In the current study, functional response properties of hV4, VO-1, and VO-2 were probed using a greater number of stimulus categories than in previous investigations (Brewer et al., 2005). We found no preference for objects versus face stimuli nor objects versus scrambled stimuli in hV4, in contrast to previous reports of object-selective responses in this region of cortex (Grill-Spector et al., 1998). Consistent with Brewer et al. (2005), we observed a preference for object relative to face stimuli in both $\mathrm{VO}$ areas. However, we observed no preference for objects versus scrambled images in $\mathrm{hV} 4, \mathrm{VO}-1$, or $\mathrm{VO}-2$, suggesting that the two $\mathrm{VO}$ areas are not part of the object-selective lateral occipital complex. This region of cortex has also been shown to be involved in texture segregation and modulated by selective attention (Kastner et al., 1998, 2000, 2001; Pinsk et al., 2004). Together, these results suggest that the $\mathrm{VO}$ areas are an intermediate visual processing stage between early visual cortex processing and higher-order object-selective cortex. It should be noted that a 
preference for scenes versus object and scrambled stimuli was observed in $\mathrm{VO}-2$, suggesting that $\mathrm{VO}-2$ may be involved in scene recognition. However, the preference for scene stimuli in $\mathrm{VO}-2$ appeared to be driven by peripheral representations because no such selectivity was observed within the fovea of VO-2.

Anterior to $\mathrm{VO}-2$, two new retinotopic areas, $\mathrm{PHC}-1$ and PHC-2, were identified. These areas may have been missed in previous investigations as a result of the smaller amount of data typically acquired in retinotopic mapping studies. We acquired approximately two to three times more data and used a phased array coil targeting ventral cortex to obtain polar angle maps. The unusually large amount of data necessary to reveal polar angle representation in this part of cortex may be attributable to neurons with large RFs, as suggested by the strong bias toward peripheral representations in the PHC areas. This bias toward peripheral eccentricities is also consistent with previous studies describing a systematic representation of eccentricity across ventral visual cortex with preference for peripheral visual field locations in PHC and preference for foveal locations in lateral occipital cortex (Levy et al., 2001; Hasson et al., 2002; Malach et al., 2002). However, our and others' (Brewer et al., 2005; Larsson and Heeger, 2006) findings of discrete foveal clusters in addition to mirror-reversing polar angle representations in ventral visual cortex are not compatible with an organizing principle based on eccentricity biases alone (Levy et al., 2001; Hasson et al., 2002; Malach et al., 2002).

Our findings do lend support to the hypothesis that visual cortex is organized at a large scale into a number of clusters that share common functional response properties (Wandell et al., 2005, 2007) (for related concepts, see Kaas and Catania, 2002). According to this account, a cluster comprised several maps with parallel eccentricity representations that share a fovea. Within a cluster, maps can be delineated on the basis of reversals in polar angle representation. Importantly, maps that belong to a cluster are characterized by similar functional computations to mediate common perceptual processes. As summarized above, the PHC areas fulfill all of these criteria, and therefore we suggest that PHC-1 and PHC-2 form a cluster that processes object information related to processing of scene stimuli. The PHC cluster adds to a growing list of functional clusters that have been identified in visual cortex: a posterior cluster comprising early visual areas V1-V3, the LO cluster comprising LO-1, LO-2, and possibly hV4 (on the basis of common response properties related to shape information), the VO cluster comprising VO-1 and VO-2 (involved with color processing), and the MT (middle temporal area) cluster (involved with motion processing). Other clusters such as the V3A/B cluster and the posterior intraparietal sulcus (IPS) cluster (IPS-0/1) need to be better defined in terms of their functional characteristics before determining whether they are consistent with the cluster hypothesis. Determining whether regions within a cluster share response properties can be difficult given that many of these clusters and areas are involved in multiple visual functions that we have only begun to define. For example, IPS-0/1 share a common fovea as well as common response properties with respect to motion and object selectivity but deviate with respect to the representation of high-level object information that is present in IPS-1 but not in V7/IPS-0 (Konen and Kastner, 2008a,b). However, the cluster hypothesis provides an overall useful and straightforward framework to characterize the large-scale organization of the visual system further.

PHC-1 and PHC-2 were found to heavily overlap with the functionally defined PPA (Epstein and Kanwisher, 1998). The PPA has been shown to respond strongly to spatial layouts such as buildings, landmarks, rooms, tabletop scenes, and even "scenes" made out of LEGO blocks (Aguirre et al., 1998; Epstein and Kanwisher, 1998; Epstein et al., 1999) (for review, see Epstein, 2008). The PPA has been shown to respond more strongly to contralateral than to ipsilateral stimuli (MacEvoy and Epstein, 2007), consistent with our findings of visual field maps in the PHC areas that represent mainly contralateral space. Furthermore, the PPA has been shown to respond more strongly to scene stimuli in the UVF compared with foveal and LVF locations (Schwarzlose et al., 2008), again consistent with our findings of a UVF bias within the PHC areas.

Whatever the implications of having topographic information in ventral temporal cortex might be, the existence of spatial maps in this region of cortex complicates the traditional view of two distinct visual pathways that segregate along the dimensions of object and space representations (Ungerleider and Mishkin, 1982). At some point of neural processing, identification and location information must be integrated. One possibility is that the integration of information occurs in a common higher-order area that both areas project to, such as prefrontal cortex, as suggested by physiological studies in monkeys (Rao et al., 1997). Another possibility is that there is parallel encoding of object and location information in both pathways, suggested by the present and recent studies (Konen and Kastner, 2008a,b). Future studies will be needed to reveal the nature of this information integration within ventral temporal cortex.

\section{References}

Aguirre GK, Zarahn E, D’Esposito M (1998) An area within human ventral cortex sensitive to "building" stimuli: evidence and implications. Neuron 21:373-383.

Bandettini PA, Jesmanowicz A, Wong EC, Hyde JS (1993) Processing strategies for time-course data sets in functional MRI of the human brain. Magn Reson Med 30:161-173.

Boussaoud D, Desimone R, Ungerleider LG (1991) Visual topography of area TEO in the macaque. J Comp Neurol 306:554-575.

Brainard DH (1997) The psychophysics toolbox. Spat Vis 10:433-436.

Brewer AA, Liu J, Wade AR, Wandell BA (2005) Visual field maps and stimulus selectivity in human ventral occipital cortex. Nat Neurosci 8:1102-1109.

Chung MK, Robbins SM, Dalton KM, Davidson RJ, Alexander AL, Evans AC (2005) Cortical thickness analysis in autism with heat kernel smoothing. Neuroimage 25:1256-1265.

Cohen MS (1997) Parametric analysis of fMRI data using linear systems methods. Neuroimage 6:93-103.

Cox RW (1996) AFNI: software for analysis and visualization of functional magnetic resonance neuroimages. Comput Biomed Res 29:162-173.

Cox RW, Jesmanowicz A (1999) Real-time 3D image registration for functional MRI. Magn Reson Med 42:1014-1018.

Dale AM, Fischl B, Sereno MI (1999) Cortical surface-based analysis. I. Segmentation and surface reconstruction. Neuroimage 9:179-194.

DeYoe EA, Carman GJ, Bandettini P, Glickman S, Wieser J, Cox R, Miller D, Neitz J (1996) Mapping striate and extrastriate visual areas in human cerebral cortex. Proc Natl Acad Sci U S A 93:2382-2386.

Downing PE, Jiang Y, Shuman M, Kanwisher N (2001) A cortical area selective for visual processing of the human body. Science 293:2470-2473.

Downing PE, Chan AW, Peelen MV, Dodds CM, Kanwisher N (2006) Domain specificity in visual cortex. Cereb Cortex 16:1453-1461.

Engel SA, Rumelhart DE, Wandell BA, Lee AT, Glover GH, Chichilnisky EJ, Shadlen MN (1994) fMRI of human visual cortex. Nature 369:525.

Engel SA, Glover GH, Wandell BA (1997) Retinotopic organization in human visual cortex and the spatial precision of functional MRI. Cereb Cortex 7:181-192.

Epstein RA (2008) Parahippocampal and retrosplenial contributions to human spatial navigation. Trends Cogn Sci 12:388-396.

Epstein R, Kanwisher N (1998) A cortical representation of the local visual environment. Nature 392:598-601.

Epstein R, Harris A, Stanley D, Kanwisher N (1999) The parahippocampal place area: recognition, navigation, or encoding? Neuron 23:115-125. 
Fischl B, Sereno MI, Dale AM (1999) Cortical surface-based analysis. II. Inflation, flattening, and a surface-based coordinate system. Neuroimage 9:195-207.

Friston KJ, Frith CD, Turner R, Frackowiak RS (1995) Characterizing evoked hemodynamics with fMRI. Neuroimage 2:157-165.

Gattass R, Sousa AP, Gross CG (1988) Visuotopic organization and extent of V3 and V4 of the macaque. J Neurosci 8:1831-1845.

Gitelman DR (2002) ILAB: a program for postexperimental eye movement analysis. Behav Res Methods Instrum Comput 34:605-612.

Grill-Spector K, Malach R (2004) The human visual cortex. Annu Rev Neurosci 27:649-677.

Grill-Spector K, Kushnir T, Hendler T, Edelman S, Itzchak Y, Malach R (1998) The sequence of object-processing stages revealed by fMRI in the human occipital lobe. Hum Brain Mapp 6:316-328.

Grill-Spector K, Kushnir T, Edelman S, Avidan G, Itzchak Y, Malach R (1999) Differential processing of objects under various viewing conditions in the human lateral occipital complex. Neuron 24:187-203.

Hansen KA, Kay KN, Gallant JL (2007) Topographic organization in and near human visual area V4. J Neurosci 27:11896-11911.

Hasson U, Levy I, Behrmann M, Hendler T, Malach R (2002) Eccentricity bias as an organizing principle for human high-order object areas. Neuron 34:479-490.

Hasson U, Harel M, Levy I, Malach R (2003) Large-scale mirror-symmetry organization of human occipito-temporal object areas. Neuron 37:1027-1041.

Haxby JV, Ungerleider LG, Clark VP, Schouten JL, Hoffman EA, Martin A (1999) The effect of face inversion on activity in human neural systems for face and object perception. Neuron 22:189-199.

Horton JC, Hoyt WF (1991) The representation of the visual field in human striate cortex. A revision of the classic Holmes map. Arch Ophthalmol 109:816-824

Kaas JH, Catania KC (2002) How do features of sensory representations develop? BioEssays 24:334-343.

Kanwisher N, McDermott J, Chun MM (1997) The fusiform face area: a module in human extrastriate cortex specialized for face perception. J Neurosci 17:4302-4311.

Kastner S, De Weerd P, Desimone R, Ungerleider LG (1998) Mechanisms of directed attention in the human extrastriate cortex as revealed by functional MRI. Science 282:108-111.

Kastner S, De Weerd P, Ungerleider LG (2000) Texture segregation in the human visual cortex: a functional MRI study. J Neurophysiol 83:2453-2457.

Kastner S, De Weerd P, Pinsk MA, Elizondo MI, Desimone R, Ungerleider LG (2001) Modulation of sensory suppression: implications for receptive field sizes in the human visual cortex. J Neurophysiol 86:1398-1411.

Kastner S, DeSimone K, Konen CS, Szczepanski SM, Weiner KS, Schneider KA (2007) Topographic maps in human frontal cortex revealed in memory-guided saccade and spatial working-memory tasks. J Neurophysiol 97:3494-3507.

Konen CS, Kastner S (2008a) Two hierarchically organized neural systems for object information in human visual cortex. Nat Neurosci 11:224-231.

Konen CS, Kastner S (2008b) Representation of eye movements and stimulus motion in topographically organized areas of human posterior parietal cortex. J Neurosci 28:8361-8375.
Kourtzi Z, Kanwisher N (2001) Representation of perceived object shape by the human lateral occipital complex. Science 293:1506-1509.

Larsson J, Heeger DJ (2006) Two retinotopic visual areas in human lateral occipital cortex. J Neurosci 26:13128-13142.

Levy I, Hasson U, Avidan G, Hendler T, Malach R (2001) Center-periphery organization of human object areas. Nat Neurosci 4:533-539.

MacEvoy SP, Epstein RA (2007) Position selectivity in scene- and objectresponsive occipitotemporal regions. J Neurophysiol 98:2089-2098.

Malach R, Reppas JB, Benson RR, Kwong KK, Jiang H, Kennedy WA, Ledden PJ, Brady TJ, Rosen BR, Tootell RB (1995) Object-related activity revealed by functional magnetic resonance imaging in human occipital cortex. Proc Natl Acad Sci U S A 92:8135-8139.

Malach R, Levy I, Hasson U (2002) The topography of high-order human object areas. Trends Cogn Sci 6:176-184.

Peelen MV, Downing PE (2005) Selectivity for the human body in the fusiform gyrus. J Neurophysiol 93:603-608.

Pelli DG (1997) The VideoToolbox software for visual psychophysics: transforming numbers into movies. Spat Vis 10:437-442.

Pinsk MA, Doniger GM, Kastner S (2004) Push-pull mechanism of selective attention in human extrastriate cortex. J Neurophysiol 92:622-629.

Rao SC, Rainer G, Miller EK (1997) Integration of what and where in the primate prefrontal cortex. Science 276:821-824.

Schwarzlose RF, Swisher JD, Dang S, Kanwisher N (2008) The distribution of category and location information across object-selective regions in human visual cortex. Proc Natl Acad Sci U S A 105:4447-4452.

Sereno MI, Huang RS (2006) A human parietal face area contains aligned head-centered visual and tactile maps. Nat Neurosci 9:1337-1343.

Sereno MI, Dale AM, Reppas JB, Kwong KK, Belliveau JW, Brady TJ, Rosen BR, Tootell RB (1995) Borders of multiple visual areas in humans revealed by functional magnetic resonance imaging. Science 268:889-893.

Swisher JD, Halko MA, Merabet LB, McMains SA, Somers DC (2007) Visual topography of human intraparietal sulcus. J Neurosci 27:5326-5337.

Talairach J, Tournoux P (1988) Co-planar stereotaxic atlas of the human brain. Stuttgart, Germany: Thieme.

Tootell RB, Mendola JD, Hadjikhani NK, Ledden PJ, Liu AK, Reppas JB, Sereno MI, Dale AM (1997) Functional analysis of V3A and related areas in human visual cortex. J Neurosci 17:7060-7078.

Tyler CW, Likova LT, Chen CC, Kontsevich LL, Schira MM, Wade AR (2005) Extended concepts of occipital retinotopy. Curr Med Imag Rev 1:319-329.

Ungerleider LG, Mishkin M (1982) Two cortical visual systems. In: Analysis of visual behavior (Goodale MA, Mansfield RJW, eds), pp 549-586. Cambridge, MA: Massachusetts Institute of Technology.

Wade AR, Brewer AA, Rieger JW, Wandell BA (2002) Functional measurements of human ventral occipital cortex: retinotopy and colour. Philos Trans R Soc Lond B Biol Sci 357:963-973.

Wandell BA, Brewer AA, Dougherty RF (2005) Visual field map clusters in human cortex. Philos Trans R Soc Lond B Biol Sci 360:693-707.

Wandell BA, Dumoulin SO, Brewer AA (2007) Visual field maps in human cortex. Neuron 56:366-383. 